Ann. Biol. anim. Bioch. Biophys., I968, 8 (I), I07-I39.

\title{
GUIDE POUR LA DISSECTION ET L'IDENTIFICALION DES PRINGIPAUX MUSCLES DE LA CARCASSE CHEZ LE LAPIN
}

\author{
J. CANTIER, A. VEZINHET
}

avec la collaboration technique de E. Bouthier, C. Bressot et J. Goussopoulos

Laboratoire de Zootechnie, Centre de Recherches agronomiques du Midi,
Ecole nationale supérieure agronomique, 34-Montpellier
Institut national de la Recherche agronomique

Si la plupart des traités d'anatomie des animaux domestiques (MONTANE, BourDELle et BRESSOU, I937; Sisson et Grossman, I953 ; NICKEI, SChumMER et SEIFERLE, I954 ; SCHWARZE, I960 ; DobBERSTEIN et HOFFMANN, I964), ne traitent pas des Rongeurs, on trouve de précieux renseignements sur l'anatomie musculaire du Lapin chez un certain nombre d'auteurs : KRAUSE (I884), AlezaIs (I90I), HAaC (I903), Gerhardt (I909), Faisan (I95I), Craigie (I966).

Mais, dans le cadre des recherches que nous poursuivons sur la croissance du Lapin, il nous a paru utile de définir une méthode de dissection et d'établir un atlas musculaire.

L'esprit dans lequel cette étude a été réalisée résulte de son objectif même : permettre une facile identification des muscles, leur dissection rapide et aisément reproductible.

\section{MATÉRIEL ET MÉTHODES}

Nous avons disséqué $\mathbf{1 2 2}$ animaux des deux sexes ayant un âge compris entre neuf jours et trois ans, issus d'une souche de lapins communs sélectionnés au Laboratoire depuis i 960 et soumis à des contrôles de croissance hebdomadaires.

Le processus opératoire est le suivant : oculaires ;

- sacrification par saignée au niveau de l'artère rétinienne, après énucléation de l'un des globes

- séparation de la peau, détachée de la tunique musculaire sous-cutanée (musculi culanei, $N$. a.) en commençant par une incision à la face interne des cuisses au niveau des articulations fémorotibiales, et en poursuivant de proche en proche jusqu'à la tête;

- - enlèvement des muscles sous-cutanés et des divers panicules adipeux superficiels;

- dissection des viscères et des dépôts adipeux intra-thoraciques et intra-abdominaux, auxquels donnent accès l'ouverture de la cage thoracique, par section des cartilages costaux au niveau des articulations sterno-costales, l'incision de la paroi abdominale le long de la ligne blanche, et l'ouverture de la symphyse pelvienne;

- extirpation de l'encéphale, de l'hypophyse, du globe oculaire restant et de la langue; 
- séparation, après écartement des muscles superficiels, de la thyroïde, des parathyroïdes externes, du thymus du cou, des glandes sous-maxillaires et de la trachée artère;

- dissection individuelle de la plupart des muscles et des dépôts adipeux inter-musculaires d'une seule demi-carcasse, car il n'a pas été relevé de différence significative, sur le plan morphologique, dimensionnel et pondéral, entre les organes des demi-carcasses droite et gauche. Les muscles dont la dissection entraînerait des pertes pondérales importantes, par déshydratation notamment, sont prélevés ultérieurement en bloc : muscles de la tête (masseters excepté), muscles du larynx, sus-costaux, inter-costaux, sternaux, inter-transversaires et inter-épineux du dos et des lombes, transverse du thorax et muscles de la queue;

- nettoyage du squelette par grattage;

- tous les organes sont pesés et éventuellement mesurés, immédiatement après leur dissection.

Pour l'exécution des planches, nous n'avons retenu que les dispositions moyennes, sans envisager les variations anatomiques individuelles, d'ailleurs extrêmement réduites dans la souche qui nous a servi.

En vue de leur individualisation, les muscles sont représentés débarrassés des aponévroses qui les recouvrent ou les relient entre eux, parfois écartés, soulevés ou sectionnés afin de dévoiler les plans sous-jacents.

\section{RÉSULTATS}

Nous avons adopté les dénominations de la nomenclature officielle (les Nomina anatomica veterinaria, Hanovre, I963), les muscles du cou, du dos, et du thorax, cités dans l'ordre de leur prélèvement, étant donc imparfaitement regroupés par régions.

\section{MUSCIES DU COU ET DU TRONC \\ (MUSCULI COLLIS, DORSIS ET THORACIS)}

Le sterno-céphalique (sternomastoïdeus, fig. I, 2) est un long muscle disposé obliquement d'arrière en avant, de l'intérieur vers l'extérieur, sur le devant du cou. Son insertion céphalique s'effectue sur l'apophyse mastoïde par un tendon, en commun avec celui de la branche cléido-mastoïdienne du brachio-céphalique ; postérieurement il est fixé, avec son homologue du côté opposé, sur l'extrémité antérieure du sternum.

Les sterno-hyoïdien et sterno-thyroïdien (sternohyoïdeus et sternothyroïdeus, fig. I, 2, 3, 4, 5) sont de petits muscles allongés, rubannés, couvrant avec leurs homologues du côté opposé la face ventrale de la trachée-artère. Le sterno-hyoïdien a son insertion antérieure à la face inférieure du corps de 1'hyoïde, le sterno-thyroïdien, sous-jacent, se fixant sur le bord postérieur du cartilage thyroïde. Postérieurement, ils s'insèrent avec leurs homologues, à la pointe et sur la face interne de la première sternèbre.

Le brachio-céphalique ou mastoïdo-huméral (brachio-cephalicus, fig. I, 2) est composé de deux branches ante-claviculaires et d'une branche post-claviculaire :

- La branche cléido-mastoïdienne est fixée, à l'avant, sur 1'apophyse mastoïde, en commun avec le sterno-céphalique et a une large insertion postérieure avec la deuxième branche sur la clavicule.

- La branche trapézienne, accolée à l'omotrachélien, est recouverte, ainsi que celui-ci, par la première branche. Elle atteint, en avant, l'apophyse basilaire de l'occipital. 
- La branche deltoïđienne, appliquée sur le devant de l'épaule, est fixée antérieurement sur la clavicule, et, postérieurement, sur la crête antérieure de l'humérus, par une aponévrose commune avec les pectoraux descendant et transverse.

L'omotrachélien (omotransversarius, fig. I, 2) long muscle s'étendant de l'humérus et de l'angle de l'épaule à l'apophyse basilaire de 1'occipital, postérieurement recouvert par le bord ventral de la partie cervicale du trapèze, se fixe sur le paracromion,

Le trapèze (trapezius, fig. I, 2) est un large et mince muscle comprenant deux parties, cervicale et dorsale, reliées par une aponévrose moyenne fixée sur l'épine acromienne et sur l'aponévrose scapulaire externe. Il s'insère sur l'occipital, le raphé cervical, les apophyses épineuses des troisième à onzième vertèbres dorsales, son bord ventral étant relié aux muscles voisins et sous-jacents par de fines aponévroses.

Le rhomboìde (rhomboideus, fig. 3) présente une portion cervicale qui se fixe sur toute la longueur du raphé cervical et sur les apophyses épineuses des trois premières vertèbres dorsales, et une portion dorsale, triangulaire, s'insérant par sa base sur les apophyses épineuses des première à septième vertèbres dorsales.

Les deux portions du rhomboïde ont une insertion commune avec le releveur de l'épaule, à la face interne de l'angle dorso-postérieur de la palette scapulaire.

Le releveur de l'épaule (fig. 3) est une longue bandelette charnue, facile à distinguer du rhomboïde cervical et qui atteint, en avant, la crête externe de l'occipital.

Le grand dorsal (latissimus dorsi, fig. 2) très grand muscle triangulaire dont la large aponévrose supérieure suit l'épine des dernières dorsales et de toutes les lombaires, est formé, dans sa partie charnue, de fibres convergeant en un tendon antéro-thoracique, qui se ploie sur le bord inférieur du grand rond, de manière à passer de la face externe à la face interne de ce muscle, et s'insérer à la face interne du corps de l'humérus. Son bord postéro-ventral s'attache par une autre aponévrose sur la face externe des six ou sept dernières côtes.

Le grand dentelé du thorax (serratus anterior, fig. 3, 8) est appliqué contre la paroi thoracique; les pointes inférieures de ses sept lanières sont intriquées avec les dentelures antérieures du grand oblique. Leurs extrémités antéro-supérieures convergent vers l'angle postéro-supéro-interne du scapulum.

L'angulaire de l'épaule (levator scapulae ou serratus cervicis, fig. 3, 7) est fixé à la face interne du bord antéro-supérieur du scapulum par une extrémité commune à ses six bandelettes, qui s'insèrent par ailleurs sur les apophyses transverses des six dernières vertèbres cervicales et sur la première côte.

Les pectoraux, à ce stade de dissection, sont seuls à maintenir le membre antérieur au tronc et sont relativement délicats à dissocier, étant donné leur intrication.

Le pectoral descendant (fig. $I, 2$ ) de couleur foncée, est un long parallélogramme fixé, d'une part sur la moitié antérieure de la première sternèbre, $d^{\prime}$ autre part sur la crête antérieure de l'humérus et la gaine du biceps brachial, avec la branche deltoïdienne du brachio-céphalique et le pectoral transverse.

Le pectoral transverse (fig. I) est un large muscle plat se fixant aux quatre premières sternèbres ; il se termine, par sa partie antérieure, à la crête antérieure de 
1'humérus, avec la branche deltoïdienne du brachio-céphalique et le pectoral descendant, et par sa partie postérieure, en un fascia très mince, étalé, en dedans đu membre, sur l'aponévrose anté-brachiale.

Le pectoral ascendant (pectoralis profondus, pars humeralis, fig. I) dont les fibres antérieures sont recouvertes par le pectoral transverse, s'insère sur le sternum, de la troisième à la dernière sternèbre, et rejoint, dans la région supérieure du bras, le trochin, le tendon d'origine du coraco-brachial et l'aponévrose d'enveloppe du tendon supérieur du biceps. Il est formé de deux portions distinctes, l'antérieure étroite étant en partie recouverte par la postérieure.

Le pectoral scapulaire (pectoralis profondus, pars praescapularis, fig. I, 2, 3) muscle mince et triangulaire dont les fibres partent des deux premières sternèbres, convergent en dehors, et croisent la clavicule en dessous en y prenant quelques attaches, et se terminent, après inflexion sur l'angle de l'épaule, à l'aponévrose couvrant le sus-épineux et se confondant avec l'aponévrose scapulaire externe.

Le pectoral accessoire du pectoral ascendant est un faisceau étroit, plaqué sur la face interne du pectoral scapulaire, qui se rend de la première sternèbre à 1'acromion et, par une aponévrose, au trochiter; sa dissection libère complètement le membre antérieur du tronc.

Le petit dentelé antérieur (serratus posterior superior, fig. 4, 8) est un petit muscle étroit et mince, allongé sur la partie supérieure des troisième à neuvième côtes à la face externe desquelles il s'insère ; son bord dorsal se continue par une aponévrose jusqu'aux apophyses épineuses des vertèbres dorsales et son extrémité antérieure se termine en un frêle faisceau croisant le splenius par-dessus, jusqu'au raphé cervical auquel il se fixe.

Le petit dentelé postérieur (serratus posterior inferior, fig. 4, 8) prolonge en arrière le précédent, jusqu'à la dernière côte.

Le droit du thorax (sternalis brutorum, fig. 5, 8) est un petit faisceau aplati, plaqué à la face inféro-externe des deux premières côtes, au-dessous des scalènes, et dirigé obliquement de haut en bas et d'avant en arrière.

Le splénius (splenius cervicis et capitis, fig. 3, 4, 7) s'attachant en avant sur la crête mastoïdienne, en haut sur le raphé cervical et par sa languette inférieure sur l'atlas, s'insère postérieurement sous le long dorsal, dans la gouttière formée par les apophyses épineuses et transverses des deux dernières vertèbres cervicales et de la première vertèbre dorsale.

Le grand complexus (complexus major, fig. 5) est fixé en haut sur le raphé cervical qu'il longe sur toute sa longueur, et en avant sur la protubérance occipitale ; en arrière il se termine par des faisceaux dont les insertions rachidiennes, en partie cachées par le transversaire du cou et les parties antérieures du long dorsal et du multifide dorso-lombaire, se font par des petites languettes aponévrotiques au niveau des apophyses transverses des trois premières vertèbres dorsales pour la branche dorsale, et des apophyses articulaires des trois dernières vertèbres cervicales pour la branche ventrale.

Le petit complexus (complexus minor, fig. 5) s'insère sur l'apophyse mastoïde du temporal et sur l'apophyse transverse de l'atlas en avant, et se termine en arrière au niveau de l'apophyse articulaire de la quatrième vertèbre cervicale. 
Le transversaire du cou (longissimus cervicis, fig. 5, 6) est un muscle étroit, logé dans la gouttière formée par le multifide dorso-lombaire et le long épineux, fixé par des fibres tendineuses sur les apophyses transverses des vertèbres cervicales et des quatre premières vertèbres dorsales.

Le long du cou (longus colli, fig. $x, 7$ ) recouvre, avec son homologue du côté opposé, la face ventrale du corps des vertèbres cervicales et des six premières dorsales. Il est formé d'une succession de faisceaux tendineux, dont l'antérieur est fixé au tubercule inférieur de l'atlas par un tendon qu'il partage avec son homologue du côté opposé, le postérieur à la face inférieure du corps des six premières vertèbres dorsales, les autres faisceaux allant d'une vertèbre cervicale à la suivante.

Le grand droit antérieur de la tête (longus capitis, fig. 6, 7) est fixé sur les apophyses transverses des troisième, quatrième et cinquième vertèbres cervicales par des languettes charnues et en avant sur les empreintes du corps du sphénoïde et 1'apophyse basilaire de l'occipital.

Le petit droit antérieur de la tête (rectus capitis anterior, fig. $4,5,6,7)$ est un petit muscle prismatique, accolé au côté externe du précédent qui le recouvre légèrement ; il s'insère sur l'arc ventral de l'atlas et en avant sur le corps du sphénoïde et l'apophyse basilaire de l'occipital.

Le grand oblique de la tête (obliquus capitis inferior, fig. 6) disposé dans une direction obliquement descendante, il s'attache par son extrémité postéro-supérieure sur le flanc de l'apophyse épineuse de l'axis et par son extrémité antéro-inférieure sur la face supérieure de l'aile de l'atlas.

Le petit oblique de la tête (obliquus capitis superior, fig. 6) est couché dans tune direction ascendante de l'aile de l'atlas à l'occipital et à l'apophyse mastoïde du temporal.

Le grand droit postérieur de la tête (rectus capitis posterior major, fig. 6) est allongé de la pointe postérieure de l'apophyse épineuse de l'axis jusqu'à l'occipital où il s'insère sous l'attache du grand complexus.

Le droit postérieur moyen (rectus capitis posterior medius, fig. 6), fixé postérieurement à la pointe antérieure de 1'apophyse épineuse de l'axis, ce muscle triangulaire prend en avant une large insertion sur la crête mastoïdienne et la face nuchale de la tête.

Le petit droit postérieur de la tête (rectus capitis posterior minor, fig. 6), muscle triangulaire appliqué sur la capsule de l'articulation occipito-atloïdienne, attaché à son origine à la face supérieure de l'atlas, il se termine à la face nuchale de l'occipital, sous l'insertion du grand droit.

Le cervical ascendant (iliocostalis cervicis, fig. 3, 4, 5, 6, 7) est formé de quatre ou cinq faisceaux successifs et chevauchants, s'insérant sur les apophyses transverses cervicales, l'antérieur sur l'aile de l'atlas.

Le petit droit latéral de la tête (rectus capitis lateralis, fig. 4, 5, 6), petit muscle appliqué sur le côté de 1'articulation atloïdo-occipitale s'attache sur l'atlas en dehors du petit droit antérieur, et par ailleurs sur la face interne de l'apophyse styloïde de l'occipital.

L'épineux du cou (spinalis cervicis, fig. 6) prend origine sur les apophyses 
épineuses des trois premières vertèbres dorsales, au-dessus des attaches du multifide dorso-lombaire ; il se termine sur les apophyses épineuses des cinq dernières cervicales.

Le transversaire épineux du cou ou multiflde du cou (multifidus cervicis, fig. 6) formé de faisceaux attachés par leur extrémité inféro-postérieure sur les tubercules articulaires des cinq dernières cervicales et des 2 premières dorsales et par leur extrémité supéro-antérieure sur les sixième, cinquième, quatrième, troisième et deuxième apophyses épineuses de la même région.

Les intertransversaires du cou (intertransversarii cervicis, fig. 4, 5, 6) sont de petits faisceaux fortement tendineux disposés en deux séries convergentes vers la tête, logées dans les excavations latérales comprises entre les apophyses transverses et les apophyses articulaires des vertèbres cervicales; il n'en existe pas entre l'atlas et l'axis.

Les scalènes (scaleni, fig. 3, 4, 5, 6, 7,8) comprennent, chez le Lapin :

- le scalène supra-costal, muscle triangulaire étalé des troisième, quatrième et cinquième vertèbres cervicales sur les apophyses transverses desquelles il prend origine par trois pointes tendineuses, jusqu'à la cinquième côte; il se divise dans sa partie postérieure en trois branches plaquées à la face externe des cinq premières côtes ;

- le scalène de la première côte qui se fixe sur l'apophyse transverse de la cinquième vertèbre cervicale d'une part, sur le bord antérieur de la première côte d'autre part.

Le long costal (longissimus costarum, fig. 5,6 ) est un bourrelet musculaire de coloration rouge foncé, strié de lignes tendineuses obliques et disposé longitudinalement à la face externe de la partie la plus convexe des côtes ; ses faisceaux naissent et se terminent successivement sur la face externe des côtes après en avoir croisé chacun deux ou trois. Le postérieur part du bord externe de la face inférieure du long dorsal au niveau des onzième et douzième côtes au-delà desquelles les deux muscles sont confondus ; la languette tendineuse de l'antérieur va jusqu'à l'apophyse transverse de la dernière cervicale.

Le long dorsal (longissimus dorsi, fig. 3, 4, 5, 6, 8) est un long et puissant muscle dont la partie antérieure s'insère sur les apophyses transverses des quatre dernières cervicales et la tubérosité des premières côtes. Il occupe postérieurement la gouttière formée par les apophyses transverses et les apophyses mamillaires lombaires et s'attache sur la crête, la face interne et les deux angles supérieurs de l'ilium.

Le long épineux (spinalis dorsi, fig. 5, 6) accolé dorsalement au précédent, a ses fibres antérieures fixées sur les apophyses articulaires des quatre dernières cervicales. Vers l'arrière, il est plaqué contre les apophyses épineuses dorsales, puis logé dans la gouttière formée par les apophyses mamillaires et les apophyses épineuses, ses parties lombaire et sacrée étant formées de faisceaux tendineux chevauchants fixés sur ces apophyses.

Le multifide dorso-lombaire ou tranversaire épineux dorso-lombaire (multifidus spinae, fig. 6) forme un bourrelet plus compact et nettement distinct par sa coloration du long épineux, plaqué sur la ligne des apophyses mamillaires. Ses attaches 
se font sur les apophyses épinetuses, les apophyses transverses, les apophyses mamillaires des quatre dernières vertèbres cervicales, des dorsales, des lombaires et des sacrées.

\section{MUSCLES ABDOMINAUX (MUSCULI ABDOMINIS)}

Étroitement plaqués les uns contre les autres, ils sont enlevés ensemble ; ce sont :

L'oblique externe de l'abdomen (obliquus externus abdominis, fig. $2,3,5,8$ ) ; le plus superficiel des abdominaux, il comporte une portion charnue, dont les fibres ont une direction oblique vers le bas et l'arrière, et qui, par son bord antéro-supérieur, s'attache sur la face externe des côtes par une dizaine de dentelures, dont les antérieures s'entrecroisent avec les dentelures postérieures du grand dentelé ; son bord supérieur se poursuit par l'aponévrose dorso-lombaire depuis la dernière côte jusqu'à l'angle externe de l'ilium ; sa portion aponévrotique fait suite inférieurement à la portion charnue et rejoint, au niveau de la ligne blanche, celle du muscle homologue du côté opposé, tandis que, par son bord postérieur, elle correspond au pli de l'aine, de l'angle externe de l'ilium au bord antérieur du pubis, et se dédouble en arrière pour donner l'arcade crurale et l'aponévrose fémorale.

L'oblique interne de l'abdomen (obliquus internus abdominis, fig. 8) ; complètement recouvert par le précédent, ce muscle s'étend de l'angle externe de l'ilium à la ligne blanche, et du pli de l'aine à l'hypocondre. Sa portion charnue s'unit par son bord supérieur à l'aponévrose dorso-lombaire ; en avant, ses fibres s'attachent sur le bord postérieur et à la face interne des dernières côtes ; son bord postérieur atteint l'arcade crurale ; son bord inférieur et antéro-inférieur se continue par la portion aponévrotique qui s'unit à celle de l'oblique externe, pour participer à la formation de la ligne blanche, et se diviser en plusieurs languettes qui gagnent la face interne des derniers cartilages costaux.

Le droit de l'abdomen (rectus abdominis, fig. 5, 8) est une bande musculeuse comprise entre l'aponévrose des obliques et celle du transverse de l'abdomen, étendue le long de la ligne blanche depuis le pubis jusqu'à la troisième côte, et s'insérant par son aponévrose antérieure à la base du cartilage de la première côte; par son bord interne, ce muscle se fixe à la ligne blanche qui le sépare de son symétrique.

Le tranverse de l'abdomen (transversus abdominis, fig. 8, 9) forme la couche profonde des parois abdominales; son bord antéro-supérieur s'attache à la face interne des côtes asternales par des dentelures qui sont en regard des digitations du diaphragme; son bord supérieur se fixe sur l'extrémité des apophyses transverses lombaires, par un mince feuillet sinueux. Son aponévrose inférieure s'insère sur toute l'étendue de la ligne blanche, depuis l'appendice xyphoïde où elle se fixe à l'avant, jusqu'à l'arcade crurale où elle s'attache à l'arrière.

\section{MUSCLES SOUS-LOMBATRES (MUSCULI DORSIS)}

Le petit psoas (psoas minor, fig. 9) est fixé, par son tendon postérieur, au tubercule de la crête ilio-pectinée ; son bord postérieur est attaché à l'aponévrose lombo-iliaque, son bord interne et sa pointe antérieure adhérant au corps des quatre dernières vertèbres lombaires.

Annales de Biologie animale. - 1968. 
Le grand psoas (psoas major, fig. 9) s'insère par sa pointe antérieure au corps des quatre dernières vertèbres dorsales et à la face interne des quatre dernières côtes. Son bord externe s'attache par une fine aponévrose au bout des apophyses transverses des vertèbres lombaires ; son bord interne a une insertion continue sur les corps vertébraux lombaires; enfin, son attache postérieure se fait, avec celle de l'iliaque, au trochantin.

Le carré des lombes (quadratus lumborum, fig. 9) est un muscle important situé sous les précédents. Il s'étend de la huitième vertèbre dorsale à la crête iliaque et au ligament sacro-iliaque, en s'insérant, par certains de ses faisceaux, sur le corps et 1'apophyse transverse des cinq dernières dorsales, la face inférieure des dernières côtes, le corps et l'apophyse transverse de toutes les vertèbres lombaires.

\section{MUSCLES DU MEMBRE POSTÉRIEUR (MUSCULI MEMBRI PELVINI)}

A ce stade est abordée la dissection du membre postérieur qui n'est pas encore parfaitement séparé du rachis.

\section{Cuisse et bassin}

Le tenseur du fascia lata (tensor fasciae latae, fig. Io, II) recouvre la face antérieure de la cuisse; par l'intermédiaire de sa vaste aponévrose, le fascia lata, il s'attache sur la rotule et les ligaments rotuliens d'une part et au bord externe du fémur, jusqu'au troisième trochanter, d'autre part; son insertion supéro-antérieure s'effectue sur l'angle externe de 1'ilium.

Le paraméral (agitator caudae, fig. Io), situé sur la face externe de la cuisse, représenterait une portion antérieure du long vaste, nettement distincte, chez le Lapin, de la fraction postérieure constituant le biceps fémoral qui le recouvre sur ses deux tiers inférieurs. Son insertion haute se fait sur la crête du sacrum, tandis que son autre extrémité est fixée par un fort tendon sur la rotule.

Le biceps fẻmoral (biceps femoris, fig. ro) a ses attaches hautes sur la tubérosité ischiale, son angle supéro-postérieur étant recouvert par un petit faisceau superficiel du demi-tendineux, lui-même fixé par une petite aponévrose sur le ligament sacro-sciatique et l'épine sacrée. Il se termine vers le bas par une large aponévrose qui se continue par l'aponévrose jambière et lui offre une vaste insertion sur le côté externe de la jambe.

Le droit interne (gracilis, fig. II) est un muscle quadrilatère, translucide tant il est mince, qui forme le plat de la cuisse. Il se fixe par tout son bord supérieur à la symphyse ischio-pubienne en commun avec son homologue ; son aponévrose antéro-inférieure atteint le ligament rotulien interne et la face interne du tibia.

Le couturier (sartorius, fig. II) longeant le bord antéro-supérieur du droit interne, est un muscle étroit et plat, qui part de l'angle antéro-externe de l'ilium et rejoint 1'aponévrose jambière, par laquelle il se lie à l'extrémité supérieure de la face interne du tibia.

Le demi-membraneux (semi-membranosus, fig. Io, II) forme le bord postérointerne de la cuisse et la pointe de la fesse. Mis à jour après l'enlèvement du demi- 
tendineux, il a ses attaches postéro-supérieures au niveau de l'aponévrose coccygienne, de la tubérosité ischiatique et de la face interne de 1'ischium. Ses tendons inférieurs se fixent sur le condyle interne du fémur et sur l'extrémité supérieure de la tubérosité interne du tibia. Le demi-membraneux renferme dans sa masse un gros faisceau rouge foncé, conoïde, facile à isoler, auquel appartient un long tendon qui va s'insérer à l'angle postérieur de la tubérosité interne du tibia.

L,e vaste interne (vastus medialis, fig. II) a ses insertions basses sur le ligament tibio-rotulien interne et le bord interne de la rotule; vers le haut il rejoint le bord interne de la face postérieure du fémur.

Le droit antérieur (rectus femoris, fig. II, I2) est un épais cylindre dont le cône aponévrotique inférieur se fixe sur la face antérieure de la rotule; son tendon supérieur bifide se termine en avant et en dehors, sous le sourcil de la cavité cotyloïde.

Le vaste externe (vastus lateralis, fig. Io), situé à l'avant du fémur, est un muscle épais et large se terminant d'une part sur la face supérieure et le côté externe de la rotule, d'autre part au bord externe de la face postérieure du fémur, et formé de deux faisceaux associés.

Le vaste intermédiaire (vastus intermedius, fig. II, I2) est un muscle rouge foncé plaqué à la face antérieure du fémur et situé dans la gouttière formée par les vastes interne et externe. Il est constitué de faisceaux prenant naissance sur toute l'étendue de la face antérieure du fémur, son insertion inférieure sur la rotule étant commune avec le droit antérieur.

L'adducteur (adductor magnus et adductor brevis, fig. II, I2) situé en arrière, est un muscle prismatique formé, chez le Lapin, par la réunion du petit et du grand adducteur de la cuisse. Il s'insère en haut sous la face interne de l'ischium et sur la lame tendineuse impaire qui s'attache à la symphyse pelvienne.

Le pectiné (pectineus, fig. I3) au-dessus et intérieurement par rapport au précédent, est situé au fond du triangle de Scarpa et formé de deux faisceaux partant du bord antérieur et de la face inférieure du pubis. Par ailleurs, il s'attache par des tendons sur le côté interne de la partie supérieure du fémur.

Le fessier superflciel (glutoeus maximus, fig. Io) est un muscle triangulaire, plat, recouvrant la partie postérieure du fessier moyen, et se fixant par sa surface libre sur l'aponévrose fessière par laquelle il prend attache à l'angle externe de l'ilium. Postérieurement il atteint l'angle postéro-externe de l'ischium et latéralement le troisième trochanter.

Le fessier moyen (glutoeus medius, fig. ro) s'insère sur le pourtour de la fosse iliaque externe, les deux angles antérieurs de l'ilium, les ligaments sacro-iliaque supérieur et sacro-sciatique et se fixe par un gros tendon sous le sommet du trochanter.

Le fessier profond (glutoeus minimus, fig. I2) est dévoilé par le retrait du précédent, dont il se différencie par sa couleur rouge foncé et la disposition rayonnée de ses fibres, qui prennent leur origine dans la fosse iliaque externe, d'où elles convergent en un fort tendon qui se fixe sur la crête du trochanter fémoral.

Le scansorius (fig. I2, I3) est situé dans la partie inférieure de la fosse iliaque externe et recouvre le col de l'ilium. Ses fibres prennent origine au-dessous de la ner- 
vure médiane de l'ilium, se dirigent en dehors et en arrière, pour se terminer à la base du trochanter, sous l'insertion du fessier profond.

Le capsulaire de la hanche (fig. I3) est un petit faisceau retiré en même temps que le scansorius, situé entre la capsule articulaire coxo-fémorale et le scansorius. Il s'insère sur l'ilium, au-dessus du sourcil de la cavité cotyloïde, et sur la face antérieure du quart supérieur du fémur.

Le pyramidal du bassin (fig. I2) est découvert ainsi que le fessier profond, après l'enlèvement du fessier moyen; c'est un faisceau triangulaire s'insérant à la partie inférieure du sacrum, passant par la grande échancrure sciatique, et se fixant par un tendon sur la partie supéro-postérieure du trochanter, en dedans de l'insertion du fessier moyen.

- Le carré crural (quadratus femoris, fig. r2), à la face postérieure du fémur, est un muscle qui croise les fibres des obturateurs, qu'il cache en partie. Postérieurement il s'attache sur la crête de la face postérieure du fémur, un peu en dessous du trochanter, et antérieurement, sur la face inférieure de l'ischium en avant de la tubérosité ischiatique.

L'obturateur interne (obturator internus) et 1'obturateur externe (obturator externus fig. 13) sont des muscles plats disposés, le premier dans la cavité pelvienne, le second dans le bassin, de façon à obturer le trou ovalaire, sur le pourtour duquel leurs fibres se fixent. C'est ainsi que les fibres de l'obturateur interne s'insèrent sur le plancher de la cavité pelvienne à la face supérieure du pubis et de l'ischium, et à la face interne de l'ilium, ainsi que dans le fond de la fosse trochantérienne où il se termine par un tendon, en passant par la petite échancrure sciatique. Celles de l'obturateur externe ont leurs insertions à la face inférieure du pubis et de l'ischium et dans la fosse trochantérienne.

Les jumeaux du bassin (gemelli pelvis, fig. I2, I3) sont deux petits muscles qui partent du bord supéro-externe de l'ischium, suivent le tendon de l'obturateur interne et se lient à lui.

Le coccygien (coccygeus, fig. I2) est un muscle plat, mince et triangulaire, situé à la face interne du ligament sacro-sciatique et qui s'attache sur la crête supérieure de l'ischium, sur la crête sus-cotyloïdienne du coxal et sur la crête de la dernière vertèbre sacrée et des deux premières vertèbres caudales.

L'iliaque (iliacus, fig. 9, $\mathrm{r}_{3}$ ) a sa portion interne insérée sur le côté du corps de la dernière vertèbre lombaire et sa portion externe fixée sur le bord inféro-externe de l'ilium et de la crête ilio-pectinée ; il se termine, à l'autre extrémité, au niveau du trochanter.

A ce stade de la dissection le membre postérieur est détaché du rachis.

\section{Jambe}

Les jumeaux (gastrocnemius, fig. Io, II, I2) forment, à la face postérieure de la jambe, deux ventres charnus réunis à leur bord postéro-interne pour former une gouttière dans laquelle sont logés l'articulation fémoro-tibiale et les muscles profonds de la jambe. Leur tendon inférieur commun s'attache sur la pointe du calcanéum :

- le jumeau externe a son insertion supérieure sur le fémur, au-dessus du condyle externe; 
- le jumeau interne se fixe aussi sur le fémur, mais au-dessus du condyle interne.

Le perforé (plantaris et flexor digitorum brevis, fig. II, I2), situé également à la face postérieure de la jambe, est un muscle très développé, aplati, dont l'insertion supérieure se situe dans le fond de la fosse sus-cotyloïdienne, son long et fort tendon inférieur glissant dans une trochlée en arrière du calcanétum.

Le soléaire (soleus, fig. II, I2) à l'extérieur du précédent, de couleur rouge foncé, étroit et allongé, est fixé par son tendon supérieur sur un tubercule de l'extrémité supérieure du péroné, son tendon inférieur s'unissant à celui des jumeaux.

Le fléchisseur interne des phalanges (flexor digitorum longus, fig. II) prend son origine sur le tiers supérieur du bord interne du tibia ainsi qu'à la tubérosité interne et supérieure du même os, se prolonge par un long tendon, qui glisse dans une petite coulisse de la malléole interne et se poursuit sur le côté du métatarsien interne jusqu'à la première phalange interne.

Le poplité (popliteus, fig. I2) est un muscle triangulaire, court et épais, plaqué à la surface supéro-postérieure du tibia, où ses fibres prennent naissance, et derrière l'articulation fémoro-tibiale. Il se fixe par ailleurs, sur le condyle externe du fémur.

Le fléchisseur externe des phalanges ((flexor hallucis longus, fig. Io, II, I2) s'insère en haut sur la face postérieure du tibia, sur le péroné et sur le ligament interosseux qui unit ces deux os ; son tendon inférieur s'engage dans la coulisse du calcanéum pour arriver à la face plantaire du pied.

Le tibial antérieur (tibialis anterior, fig. IO, II, I2) est constitué de fibres qui s'insèrent en haut sur la surface externe de la tubérosité interne du tibia et sur le côté de la coulisse tibiale. Vers le quart inférieur du tibia, elles se réunissent en un fort et long tendon qui s'engage sous la bride tibio-tarsienne et se termine au métatarsien de l'index.

L'extenseur antérieur des phalanges (extensor longus digitorum pedis, fig. Io, II, I2), fusiforme, recouvre la face antéro-externe du tibia.' Son long et fort tendon supérieur se fixe à l'extrémité inférieure du fémur, entre le condyle externe et la trochlée; son tendon terminal inférieur passe sous les brides tibio-tarsienne puis tarsienne, et s'insère par quatre branches sur les phalanges des doigts.

Les péroniers (peronei, fig. Io, I2) sont, chez le Lapin, au nombre de quatre. Leurs corps charnus, courts, se prolongent, à partir de la fin du tiers supérieur du tibia, par de longs tendons qui glissent et s'infléchissent sur 1'extrémité antérieure du péroné ou dans la coulisse malléolaire externe, pour se diviser et se répartir ensuite sur les métatarsiens et les phalanges.

\section{MUSCLES DU MEMBRE ANTÉRIEUR (MUSCULI MEMBRI THORACICI)}

\section{Epaule}

Le deltoide (deltoideus, fig. $2,3, \mathrm{I} 4$ ) est un muscle plat et triangulaire recouvrant les deux tiers inférieurs du sous-épineux. Il est lui-même recouvert de l'aponévrose scapulaire, par laquelle il s'attache à l'épine acromienne. Sa pointe inférieure croise le paracromion en dessous et s'insère sur la crête deltoïdienne de l'humérus. 
Le sous-épineux (intraspinatus, fig. 3, I4) occupe la fosse sous-épineuse où s'attachent ses fibres qui s'insèrent également sur le cartilage de prolongement du scapulum; son fort tendon inférieur atteint le trochiter.

Le sus-épineux (supraspinatus, fig. 2, 3, I4, I5) s'insère dans toute l'étendue de la fosse sus-épineuse qu'il déborde à l'avant, sur le cartilage de prolongement du scapulum, le bord antérieur et l'angle cervical du scapulum. Il s'insère par ses branches inféro-interne et inféro-externe sur le trochin et le sommet du trochiter.

Le sous-scapnlaire (subscapularis, fig. I5) occupe la fosse sous-scapulaire dans laquelle ses fibres s'insèrent; il s'attache en outre sur le trochin par un fort tendon inférieur.

Le grand rond (teres major, fig. 3, I4, I5), en arrière du sous-scapulaire, est un muscle épais qui va de l'angle dorsal du scapulum et du cartilage de prolongement, à la face interne du corps de l'humérus où il se fixe par un tendon qu'il partage avec le grand dorsal.

Le petit rond (teres minor, fig. I4), est un muscle ayant ses attaches sur le bord postérieur du scapulum et le bas de la fosse sous-épineuse, sur le petit tubercule situé au côté externe du sourcil de la cavité glénoïde, et sur l'humérus, entre le trochiter et la crête deltoïdienne.

Le coraco-brachial (coraco-brachialis, fig. I5) est un petit muscle fusiforme qui s'insère sur le bec de l'apophyse coracoïde par un petit tendon, s'infléchit sur le tendon terminal du sous-scapulaire, et s'attache sur l'humérus un peu au-dessous de l'insertion du grand rond.

\section{Bras}

Le biceps brachial (biceps brachii, fig. I4, I5) est un muscle allongé à l'avant du bras, qui a son attache supérieure sur le tubercule sus-glénoïdien et se fixe par un fort tendon sur le côté interne du radius, au-dessous de l'articulation du coude.

Le brachial antérieur (brachialis, fig. I4) est appliqué contre l'humérus qu'il contourne partiellement de telle sorte que son insertion supérieure se situe sur la face postéro-externe de l'humérus, au-dessous de la tête articulaire, et son insertion inférieure, par un fort tendon, au niveau de 1'attache du biceps brachial.

Le long anconé (caput longum tricipitis brachii, fig. I4, I5) est un gros muscle situé en arrière de 1'humérus qui se fixe d'une part au-dessus de la cavité glénoïde, d'autre part, par son gros tendon inférieur, sur le sommet de l'olécrane.

L'anconé accessoire du grand dorsal (accessorius latissimi dorsi, fig. I5) est un petit faisceau plat qui part de l'aponévrose anté-brachiale et adhère au précédent jusqu'à son insertion inférieure.

L'anconé externe (caput laterale tricipitis brachii, fig. I4) s'insère à la face externe de 1'humérus, sur la ligne courbe qui part de la crête deltoïdienne, pour se rendre à la base de la tête articulaire, son attache inférieure se faisant sur l'olécrane.

L'anconé interne (caput mediale tricipitis brachii, fig. I5) a ses fibres qui partent de presque toute la longueur de la face postéro-interne de l'humérus et se fixe par son tendon distal sur l'olécrane.

Le petit anconé (anconoeus, fig. I4) est situé au pourtour de la fosse olécra- 
nienne, au-dessus et en dehors principalement, et se termine sur la partie antéroexterne de l'olécrane.

L'anconé profond (fig. I5), disposé à la face postérieure de l'humérus, s'attache d'une part à la base de la tête humérale, d'autre part, en avant du sommet de l'olécrane.

\section{Avant-bras}

Région antéro-interne (muscles extenseurs).

L'extenseur antérieur du métacarpe (extensor carpi radialis longus et extensor carpi radialis brevis, fig. $\mathrm{I5}$, I6) recouvre la face antérieure de la moitié supérieure du radius, s'insère sur l'épicondyle de l'humérus, tandis que les deux branches de son tendon distal se rendent respectivement à l'extrémité des métacarpiens de l'index et du médius.

L'extenseur oblique du métacarpe (abductor pollicis longus et extensor pollicis brevis, fig. I5, I6) suit 1'interligne radio-cubital, se fixe sur le bord externe du radius et a son tendon inférieur qui croise obliquement et en dessus les tendons du précédent pour atteindre l'extrémité supérieure du métacarpien du pouce.

Le cubital externe (extensor carpi ulnaris, fig. I6) très grêle extenseur chez le Lapin, s'attache sur le sommet de l'épicondyle et par son tendon inférieur sur l'extrémité proximale du cinquième métacarpien, après avoir pris attache sur l'os suscarpien.

L'extenseur commun des phalanges (extensor digitorum communis, fig. I6) a son corps plaqué sur la moitié proximale de la face antérieure du cubitus et s'insère sur l'épicondyle de l'humérus ; il se divise distalement en quatre branches, prolongées par autant de tendons qui gagnent respectivement la troisième phalange des quatre doigts externes.

L'extenseur latéral des phalanges (extensor digitalis lateralis, fig. I6) est clivé sur toute sa longueur en deux muscles très grêles, s'attachant sur le ligament externe de l'articulation du coude, sur l'épicondyle de 1'humérus et sur la surface inféroexterne du cubitus : l'extenseur propre du quatrième doigt et l'extenseur propre du cinquième doigt, dont les tendons gagnent respectivement les premières phalanges des quatrième et cinquième doigts.

L'extenseur du pouce et de l'index (extensor pollicis longus et extensor indicis proprius, fig. r6) est un très petit muscle, situé sous le précédent, dont l'insertion supérieure s'effectue en commun avec celle de 1'extenseur oblique du métacarpe, et dont le tendon distal, filiforme, après avoir franchi la face antérieure du carpe, croise en dessous celui de l'extenseur commun des doigts, pour aller du côté interne et se diviser, dans la région métacarpienne, en deux branches, allant respectivement au pouce et à l'index.

Région postéro-interne (muscles fléchisseurs).

Le cubital interne (flexor carpi ulnaris, fig. $\mathrm{I} 5, \mathrm{I} 6$ ) est composé d'une portion cubitale superficielle s'insérant sur le bord postérieur de l'olécrane et d'une portion humérale profonde, plus développée, se fixant sur l'épitrochlée de l'humérus. Les 
deux parties s'unissent à leur extrémité distale, pour prendre, par leur tendon commun, insertion sur l'os sus-carpien.

Le grand palmaire ou fléchisseur interne du métacarpe (flexor carpi radialis fig. I5), petit, s'insère sur l'épitrochlée ; son tendon suit le bord interne du radius' s'engage en arrière du carpe dans une coulisse tendineuse, et se fixe à l'arrière de l'extrémité proximale du métacarpien de l'index.

Le perforé ou féchisseur superficiel des phalanges (flexor digitorum sublimus, fig. I5) s'attache proximalement sur l'épitroclée et se prolonge au niveau du carpe par un fort tendon qui se divise en quatre branches fixées respectivement à la deuxième phalange des quatre doigts externes.

Le perforant ou fléchisseur profond des phalanges (flexor digitorum profondus, fig. I5) est composé d'une importante portion humérale, divisée elle-même en trois faisceaux et fixée sur l'épitrochlée, d'une portion radiale plus petite et insérée sur le bord supéro-interne du radius, et d'une portion cubitale également faible attachée sur presque toute la longueur de la face postérieure du cubitus. L'insertion distale de ces trois portions se fait par un seul très fort tendon s'engageant dans la gaine carpienne, et se divisant en cinq branches atteignant respectivement les dernières phalanges de chaque doigt.

Le rond pronateur (pronator teres, fig. I5), qui s'insère sur l'époitrochlée et les deux tiers proximaux du bord interne du radius, est, dans sa partie distale, une mince et courte bandelette aponévrotique. 


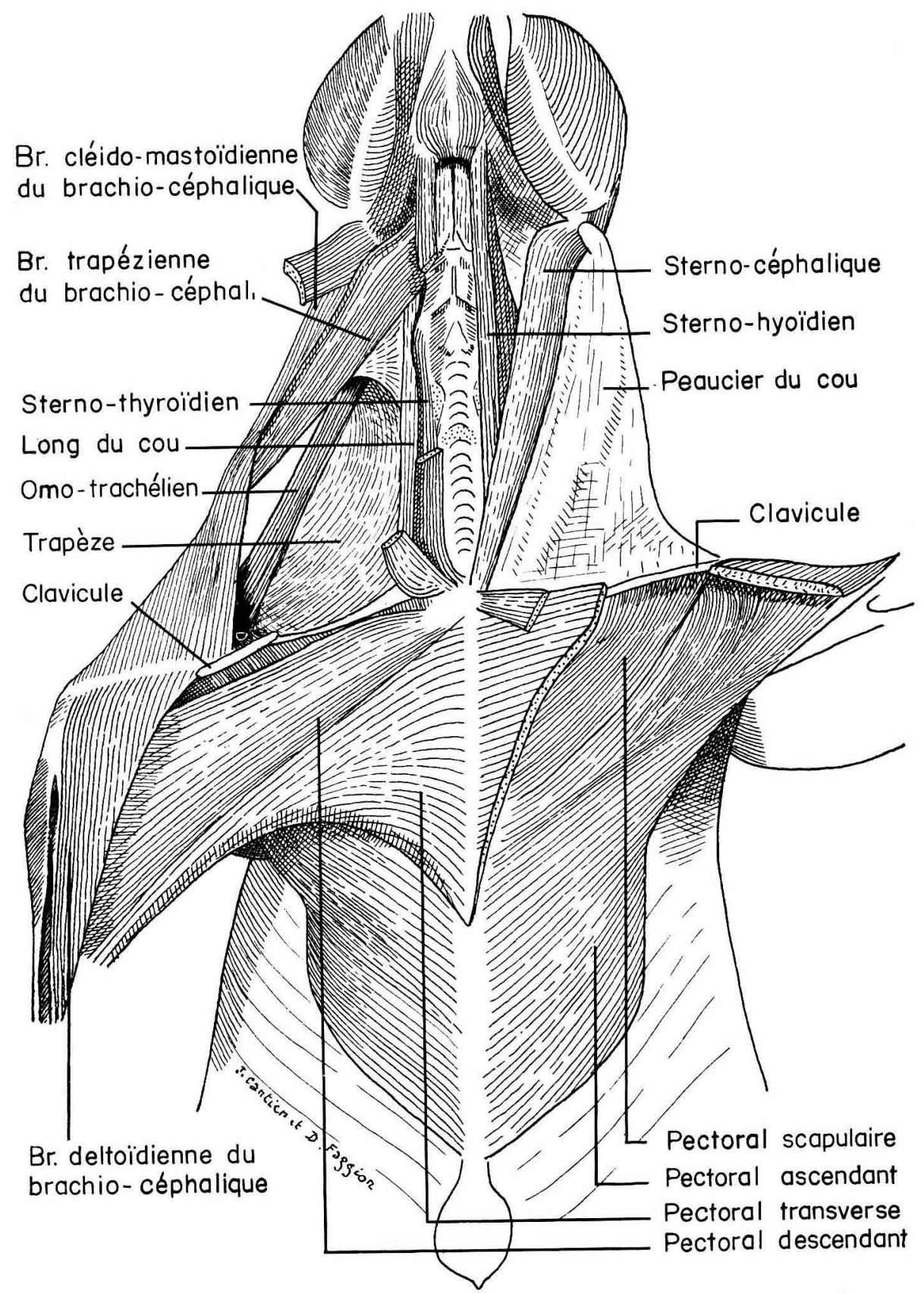

FIG. I. - Pectoraux et muscles superficiels du cou 


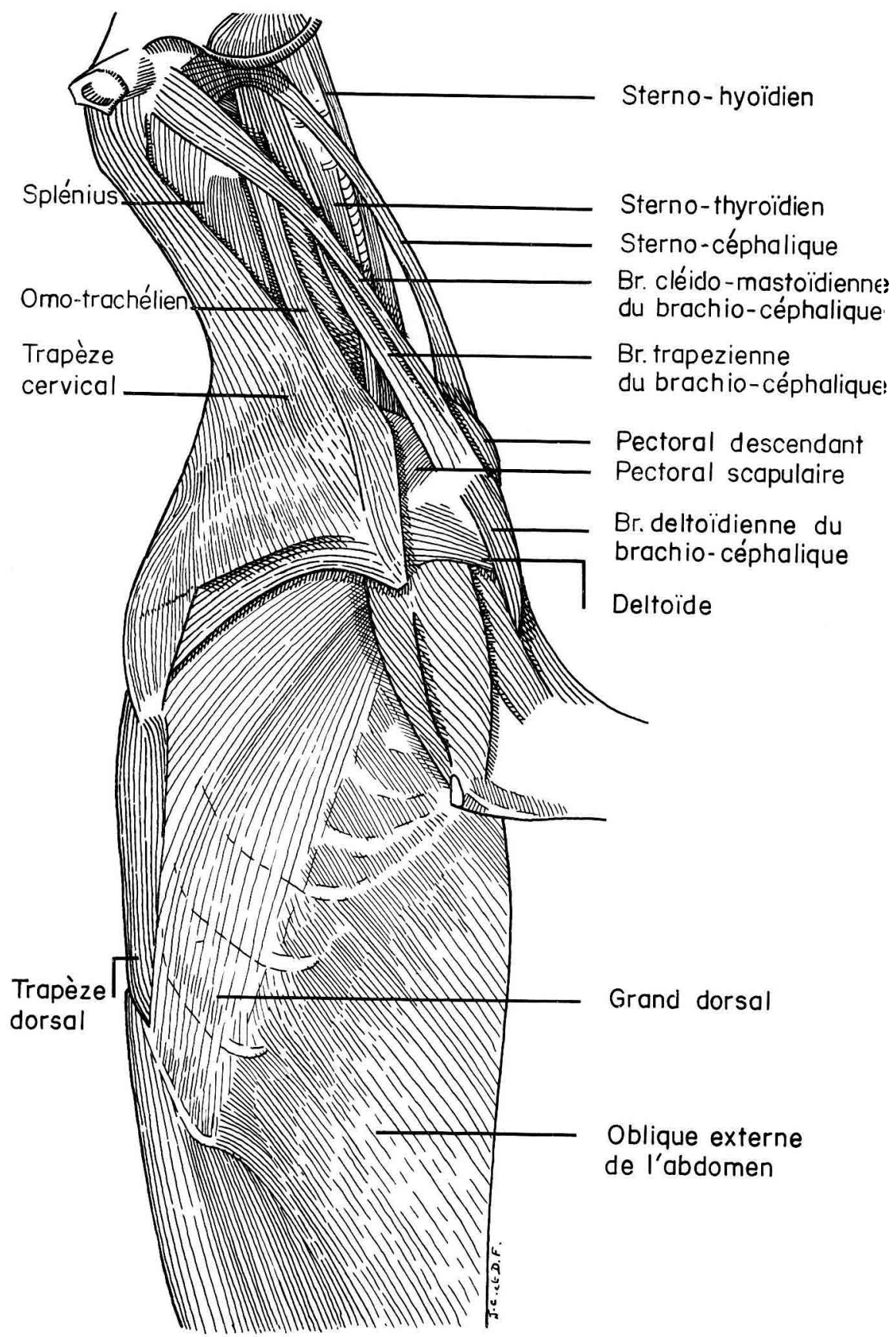

FIG. 2. - Muscles superficiels $d u$ cou 


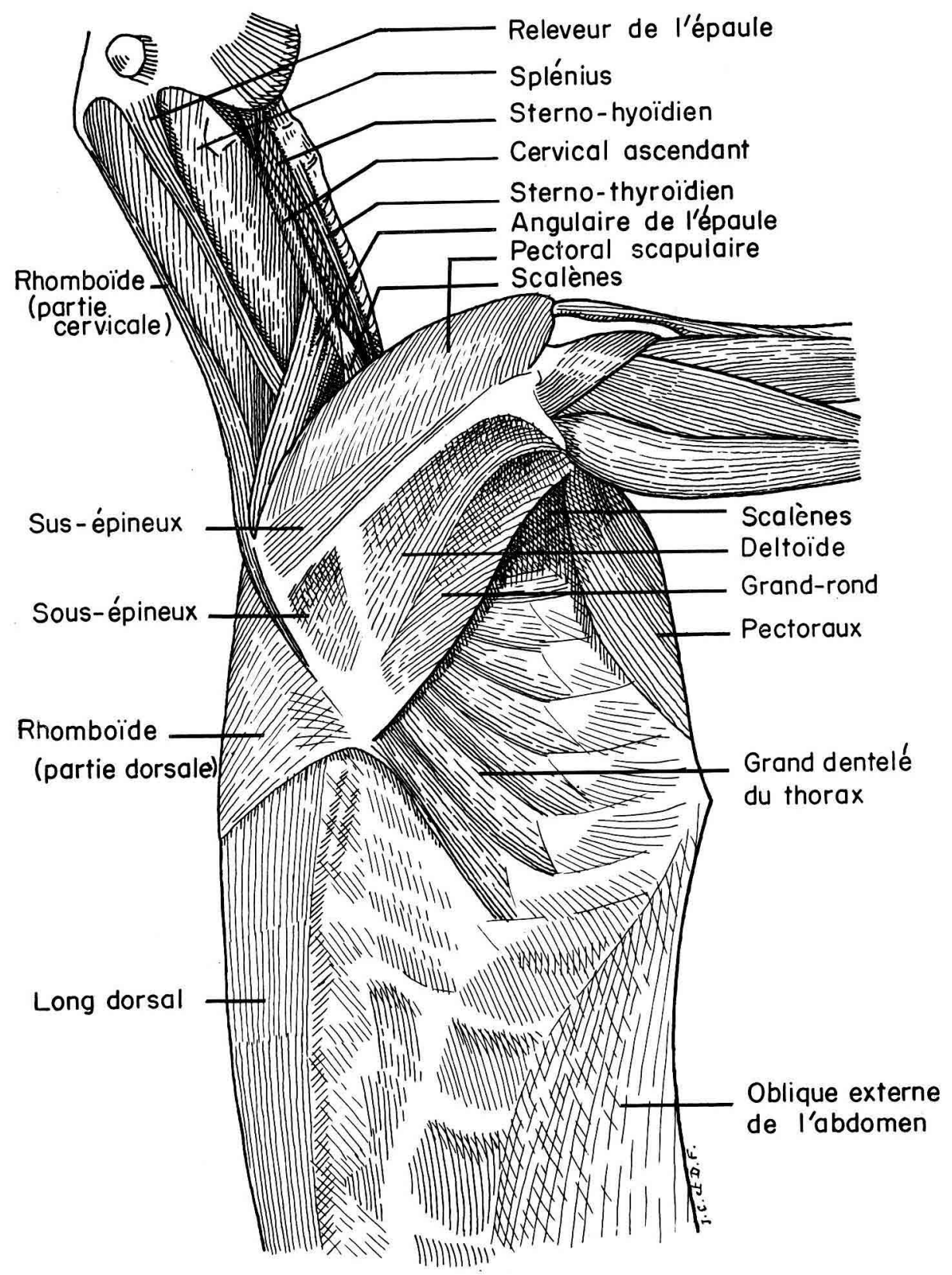

FIG. 3 . 


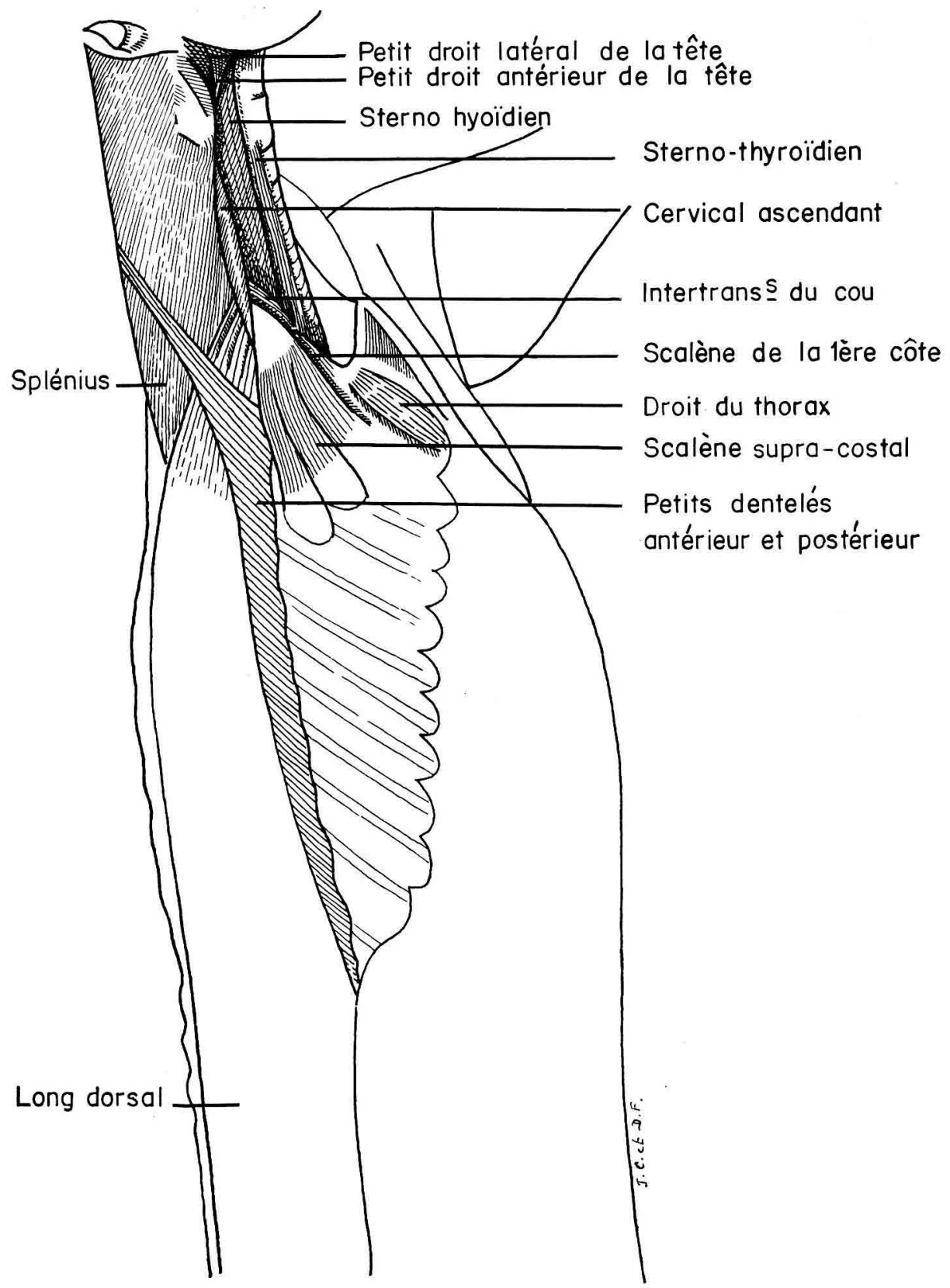

FIG. 4. 


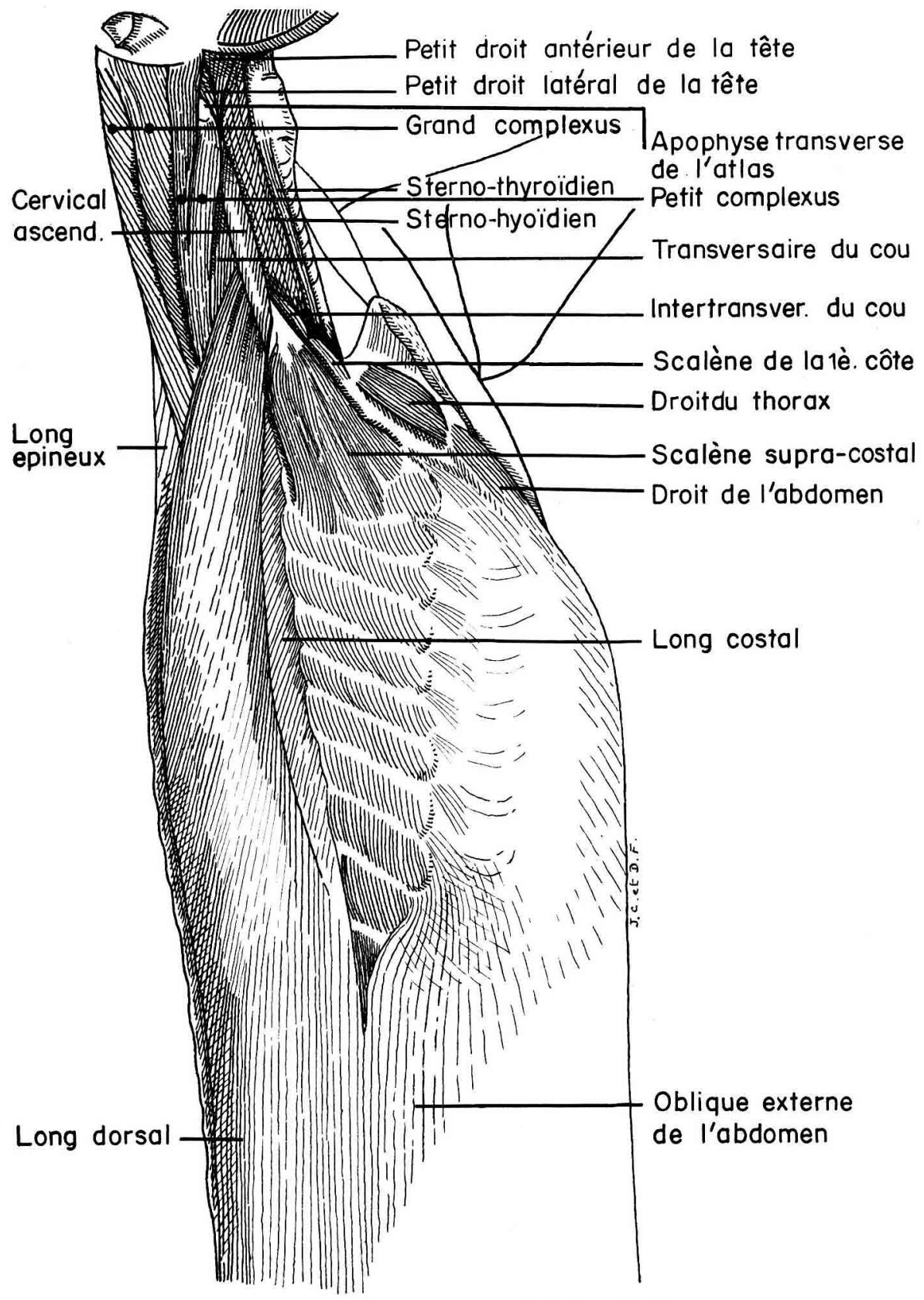

Fig. 5. - Muscles àu cou (splènius enlevê) 


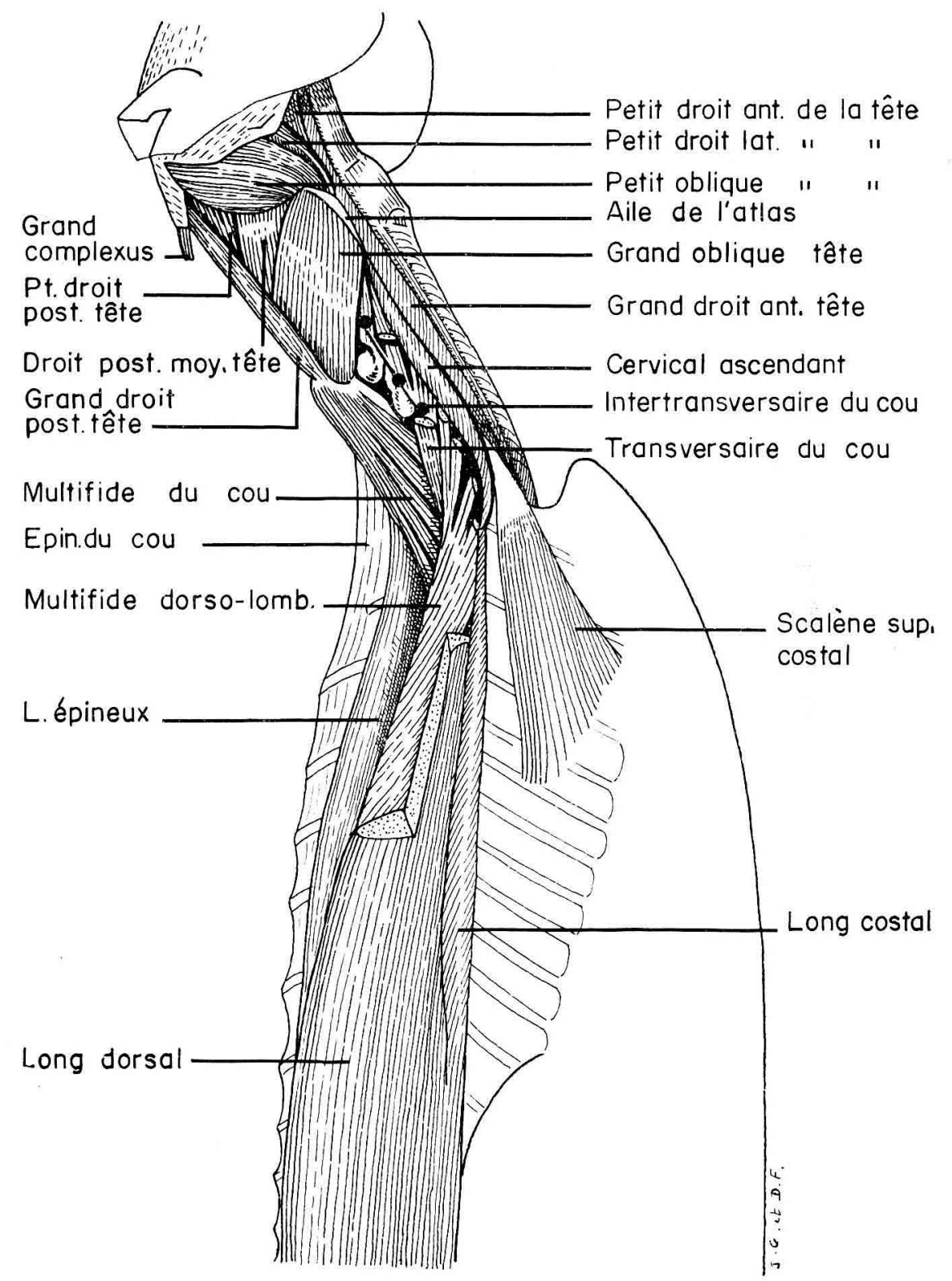

FIG. 6. - Muscles profonds du cou (splénius, grand et petit complexus enlevés) 


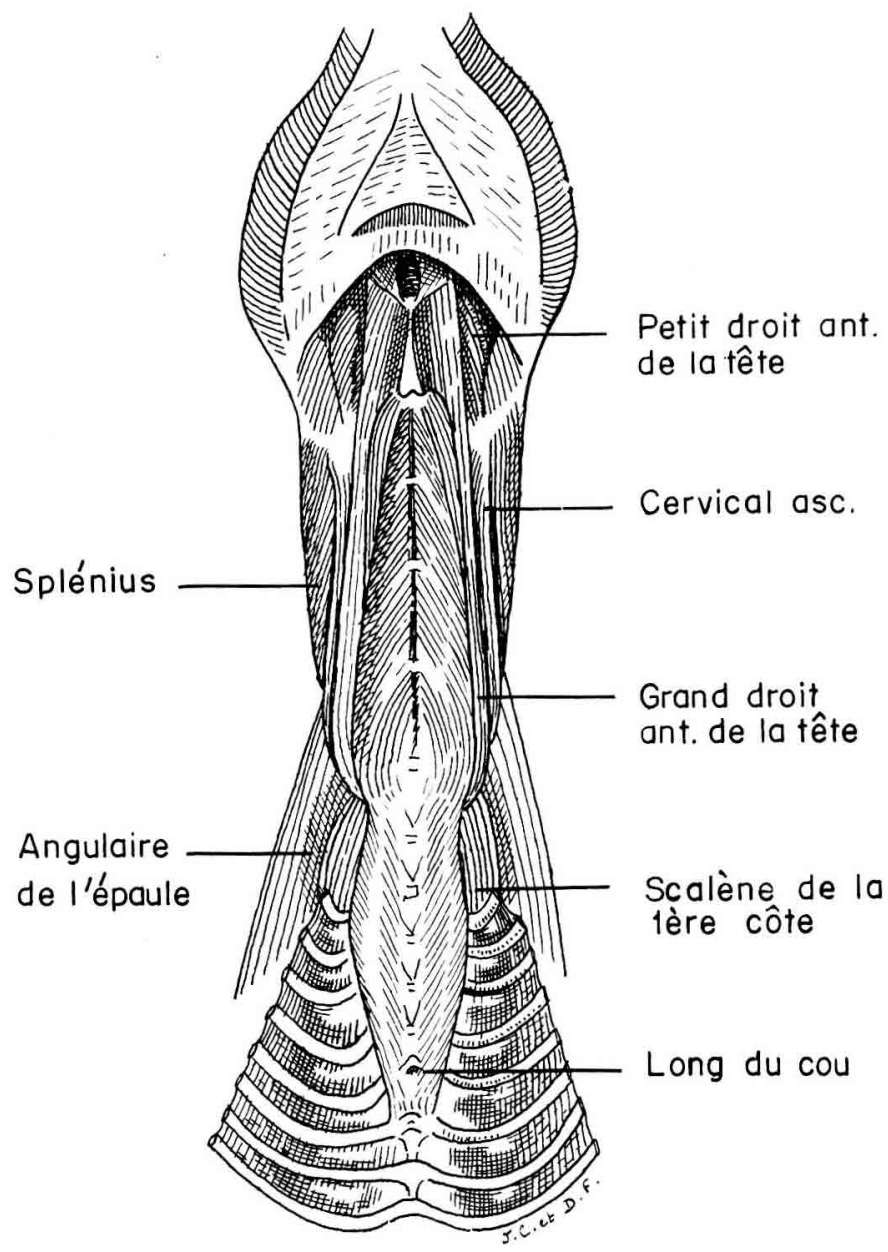

FIG. 7. - Muscles ventraux du cou 


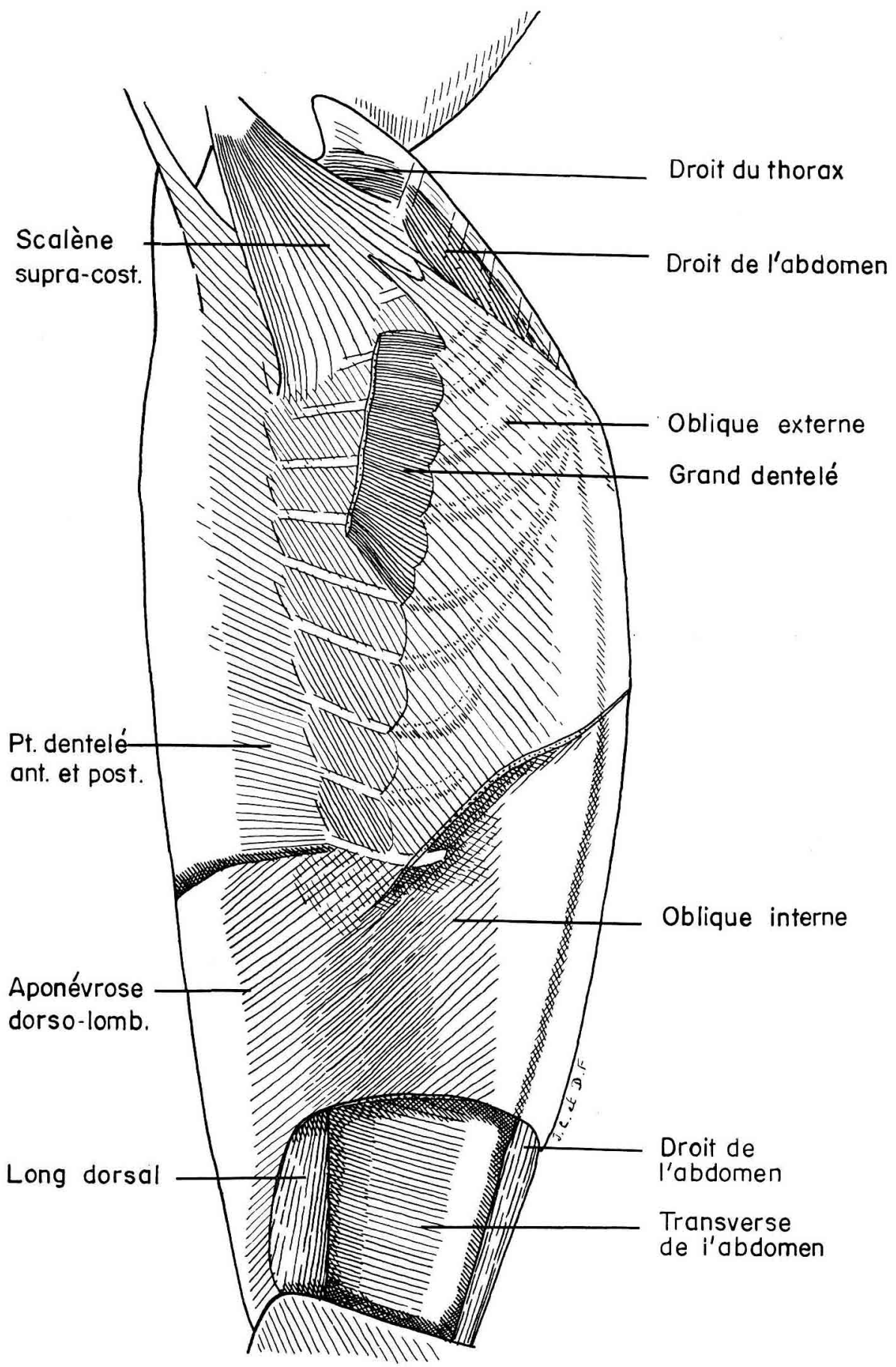

FIG. 8. - Muscles abdominaux 


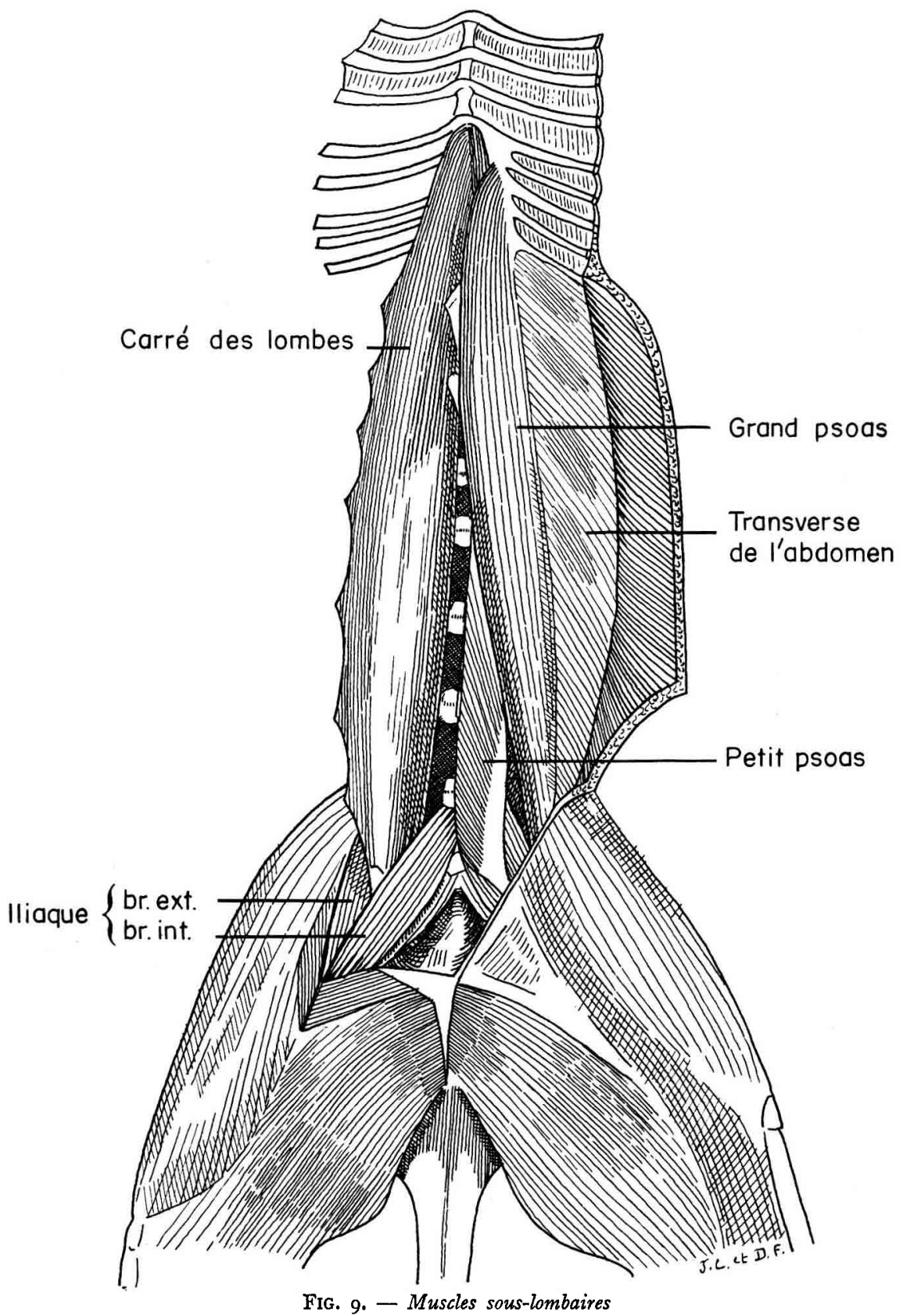




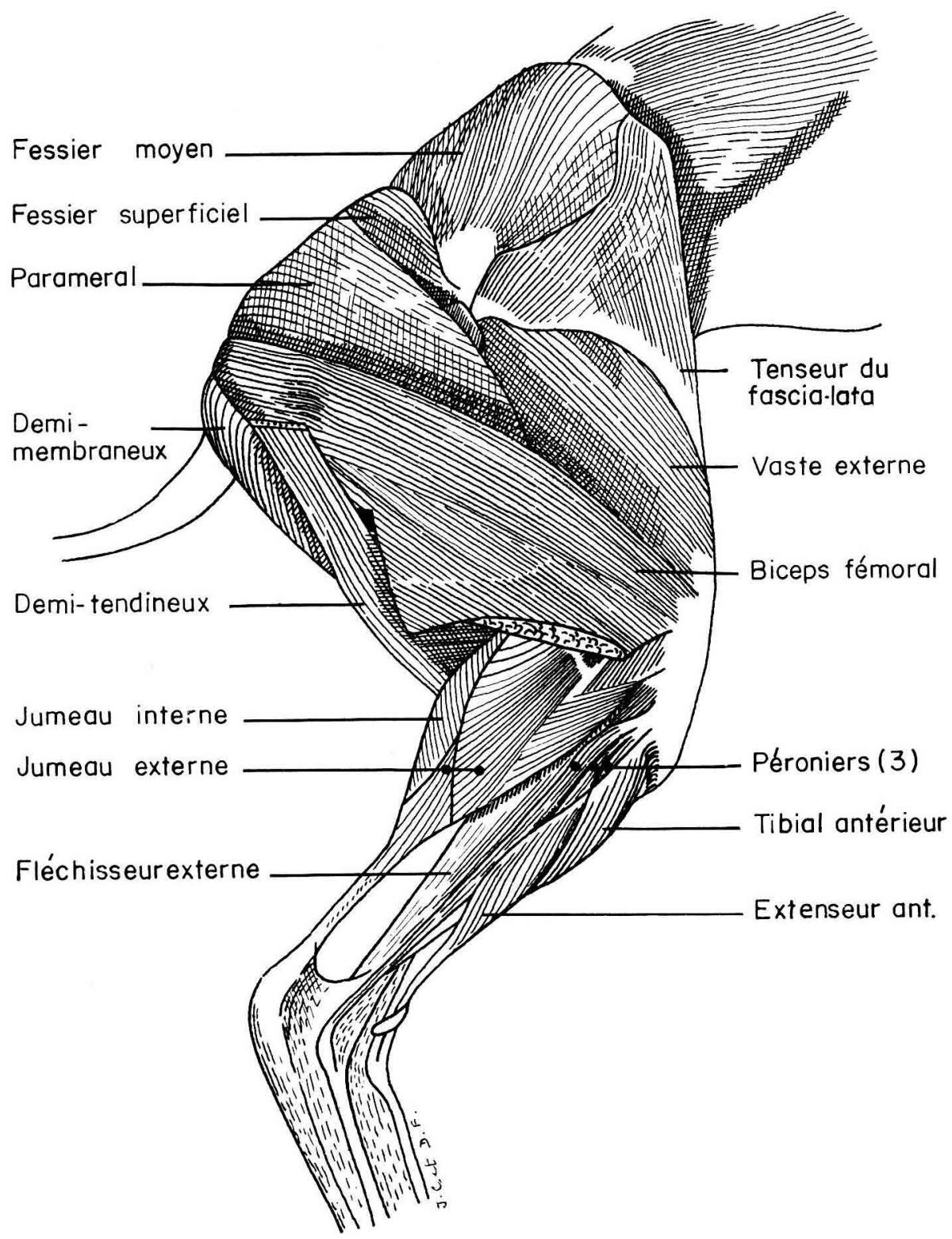

FIG. Io. - Membre postérieur, face externe 


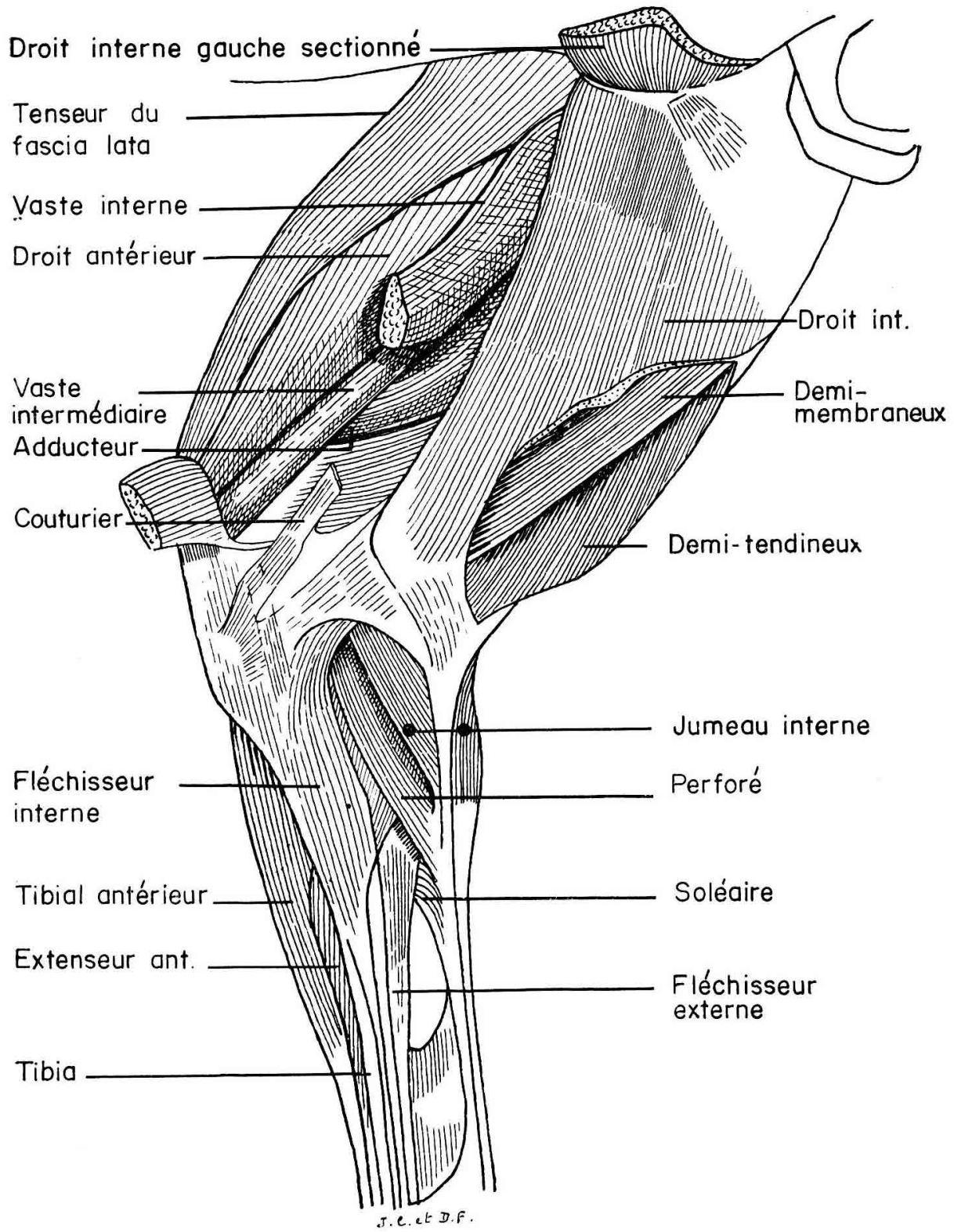

FIG. I1. - Membre postérieur, face interne 


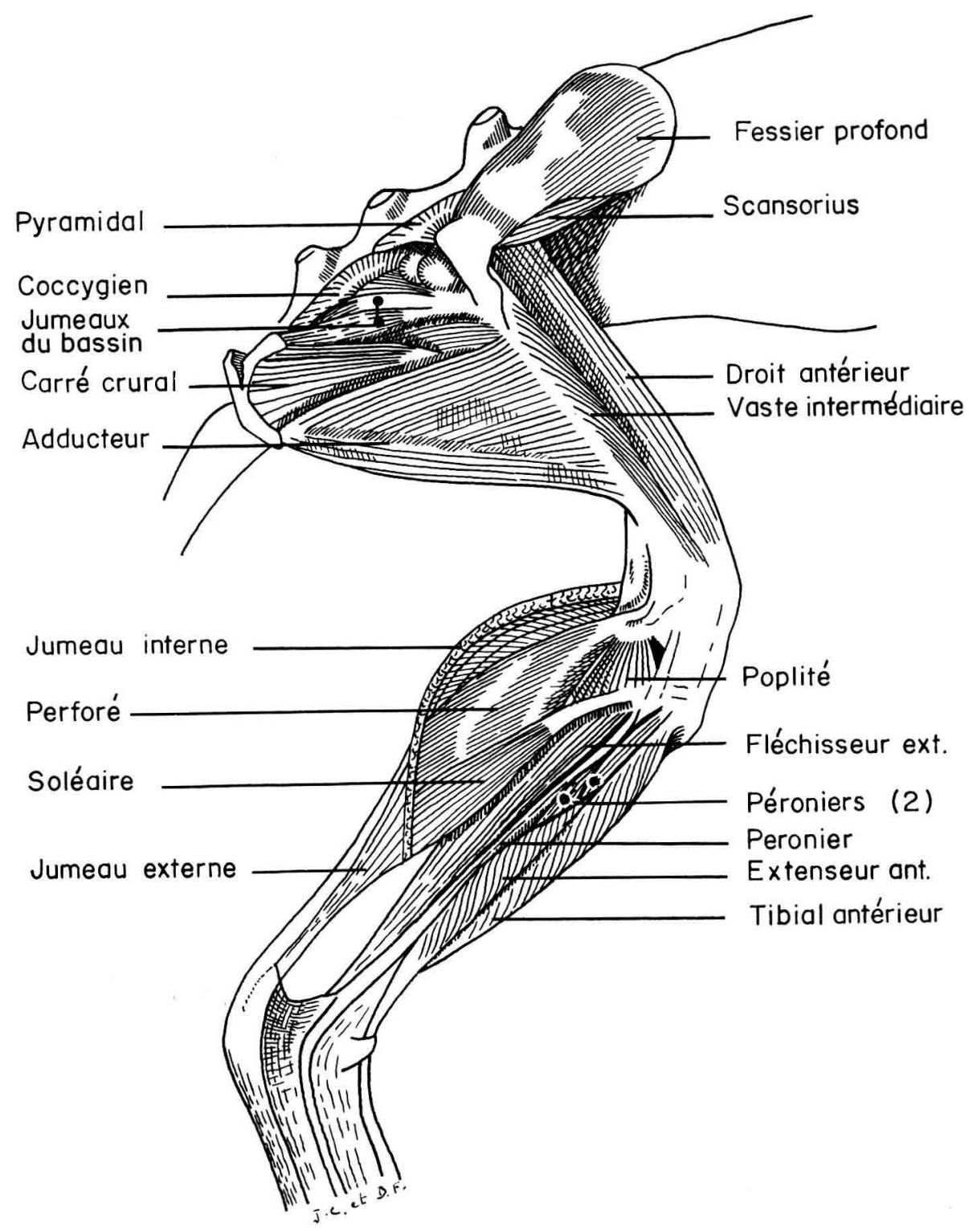

FIG. 12. - Membre postérieur, face externe (muscles profonds) 


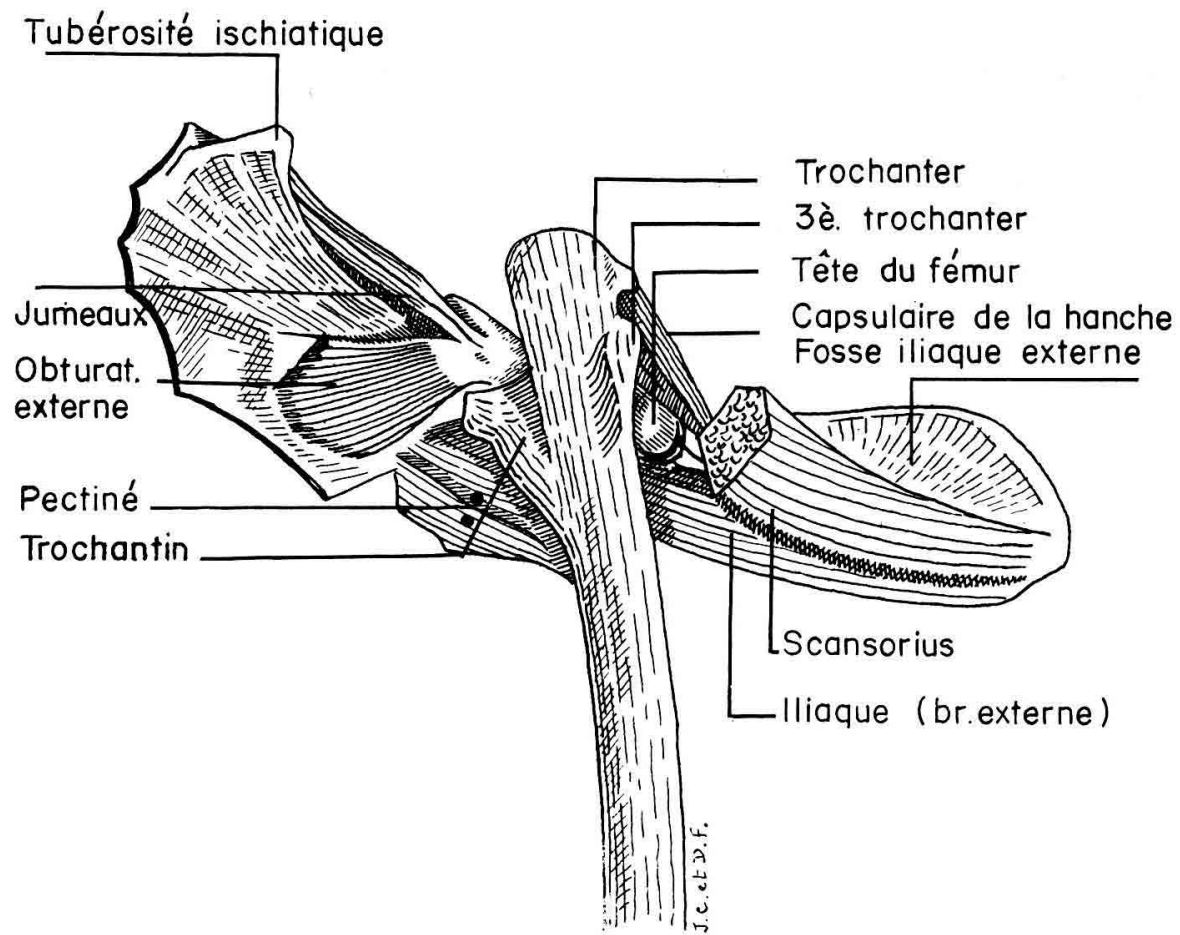

FIG. I3. - Membre postérieur, face postéro-externe (muscles profonds) 


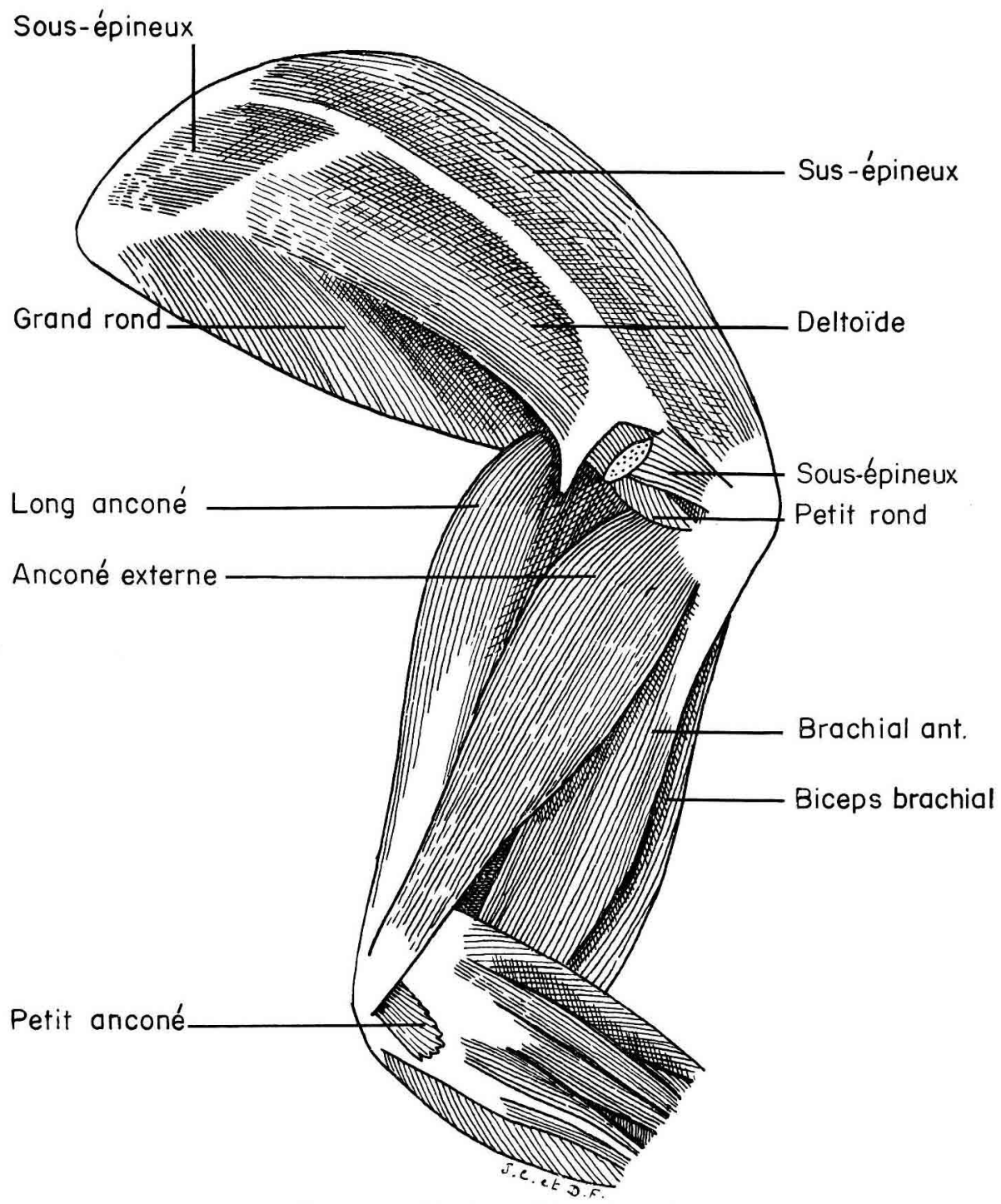

FIG. I4. - Membre antérieur, face externe 


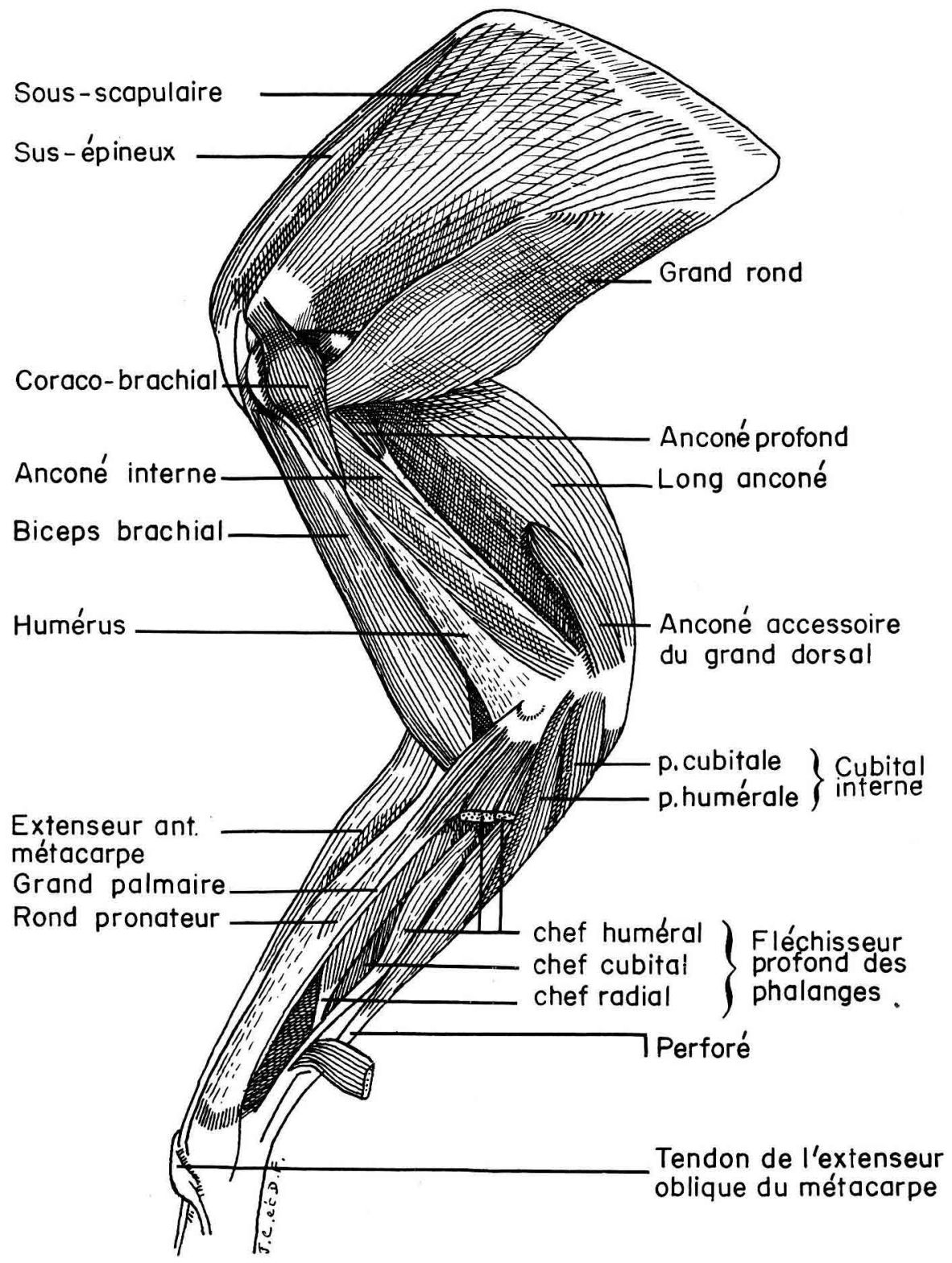

FIG. I5. - Membre antérieur, face interne 


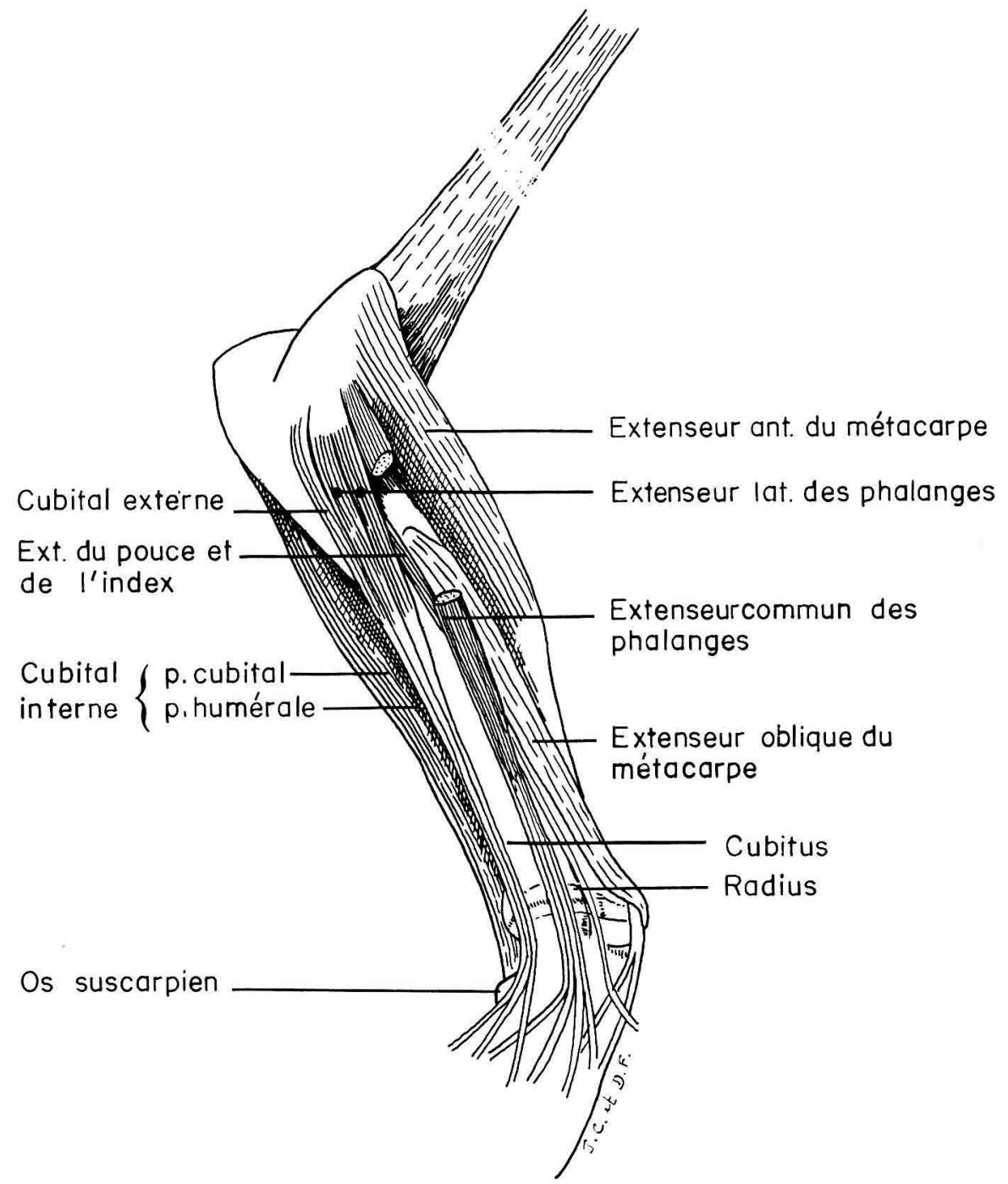

FIG. I6. - Avant-bras, face externe 
IISTE DES MUSCLES PAR ORDRE ALPHABÉTIQUE

\begin{tabular}{|c|c|c|}
\hline Nom des muscles & Figures & Pages \\
\hline Adducteur $\ldots \ldots \ldots \ldots \ldots \ldots \ldots \ldots \ldots$ & 11,12 & 115 \\
\hline Anconé accessoire du grand dorsal ........ & 15 & 118 \\
\hline 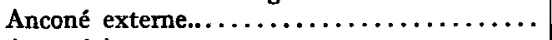 & 14 & 118 \\
\hline Anconé interne $\ldots \ldots \ldots \ldots \ldots \ldots \ldots \ldots$ & 15 & 118 \\
\hline Anconé profond $\ldots \ldots \ldots \ldots \ldots \ldots \ldots \ldots$ & 15 & $119^{\circ}$ \\
\hline Angulaire de l'épaule..$\ldots \ldots \ldots \ldots \ldots$ & 3,7 & 109 \\
\hline 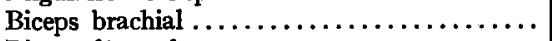 & 14,15 & 118 \\
\hline Biceps fémoral $\ldots \ldots \ldots \ldots \ldots \ldots \ldots \ldots$ & 10 & 114 \\
\hline 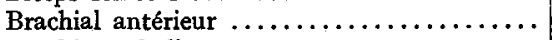 & 14 & 118 \\
\hline Brachio-céphalique.$\ldots \ldots \ldots \ldots \ldots \ldots$ & 1,2 & 108 \\
\hline 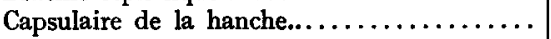 & 13 & 116 \\
\hline Carré crural $\ldots \ldots \ldots \ldots \ldots \ldots \ldots \ldots$ & 12 & 116 \\
\hline 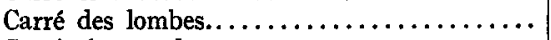 & 9 & 114 \\
\hline 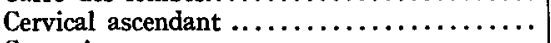 & $3,4,5,6,7$ & 111 \\
\hline Coccygien..$\ldots \ldots \ldots \ldots \ldots \ldots \ldots \ldots$ & 12 & 116 \\
\hline 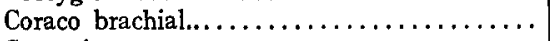 & 15 & 118 \\
\hline Couturier $\ldots \ldots \ldots \ldots \ldots \ldots \ldots \ldots \ldots$ & 11 & 114 \\
\hline Cubital externe..$\ldots \ldots \ldots \ldots \ldots \ldots \ldots$ & 16 & 119 \\
\hline 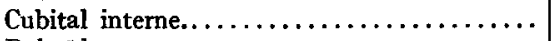 & 15,16 & 119 \\
\hline 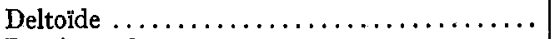 & $2,3,14$ & 117 \\
\hline 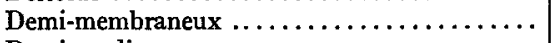 & 10,11 & 114 \\
\hline 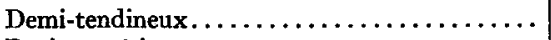 & 10,11 & 114 \\
\hline Droit antérieur $\ldots \ldots \ldots \ldots \ldots \ldots \ldots \ldots$ & 11,12 & 115 \\
\hline Droit de l'abdomen..$\ldots \ldots \ldots \ldots \ldots \ldots$ & 5,8 & 113 \\
\hline 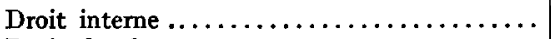 & 11 & 114 \\
\hline 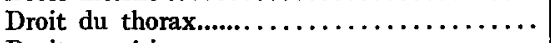 & 5,8 & 110 \\
\hline Droit postérieur moyen..$\ldots \ldots \ldots \ldots \ldots$ & 6 & 111 \\
\hline Epineux du cou $\ldots \ldots \ldots \ldots \ldots \ldots \ldots$ & 6 & 111 \\
\hline Extenseur antérieur du métacarpe......... & 15,16 & 119 \\
\hline $\begin{array}{c}\text { Extenseur antérieur des phalanges (membre } \\
\text { postérieur) } \ldots \ldots \ldots \ldots \ldots \ldots \ldots \ldots \ldots \ldots \ldots \ldots \ldots \ldots\end{array}$ & $10,11,12$ & 117 \\
\hline $\begin{array}{l}\text { Extenseur commun des phalanges (membre } \\
\text { antérieur) } \ldots \ldots \ldots \ldots \ldots \ldots \ldots \ldots \ldots \ldots \ldots\end{array}$ & 16 & 119 \\
\hline Extenseur du pouce et de l'index .......... & 16 & 119 \\
\hline $\begin{array}{l}\text { Extenseur latéral des phalanges (membre anté- } \\
\text { rieur) } \ldots \ldots \ldots \ldots \ldots \ldots \ldots \ldots \ldots \ldots\end{array}$ & 16 & 119 \\
\hline Extenseur oblique du métacarpe............ & 15,16 & 119 \\
\hline Fessier moyen $\ldots \ldots \ldots \ldots \ldots \ldots \ldots \ldots \ldots$ & 10 & 115 \\
\hline Fessier profond $\ldots \ldots \ldots \ldots \ldots \ldots \ldots$ & 12 & 115 \\
\hline Fessier superficiel $\ldots \ldots \ldots \ldots \ldots \ldots \ldots \ldots$ & 10 & 115 \\
\hline $\begin{array}{c}\text { Fléchisseur externe des phalanges (membre } \\
\text { postérieur) } \ldots \ldots \ldots \ldots \ldots \ldots \ldots \ldots \ldots \ldots\end{array}$ & $10,11,12$ & 117 \\
\hline Fléchisseur ínterne des phalanges (membre pos- & & \\
\hline térieur) $\ldots \ldots \ldots \ldots \ldots \ldots \ldots \ldots \ldots \ldots$ & 11 & 117 \\
\hline Grand complexus $\ldots \ldots \ldots \ldots \ldots \ldots \ldots$ & 5 & 110 \\
\hline Grand dentelé du thorax .............. & 3,8 & 109 \\
\hline Grand dorsal $\ldots \ldots \ldots \ldots \ldots \ldots \ldots \ldots$ & 2 & 109 \\
\hline Grand droit antérieur de la tête.......... & 6,7 & 111 \\
\hline Grand droit postérieur de la tête ......... & 6 & 111 \\
\hline Grand oblique de la tête ............... & 6 & 111 \\
\hline
\end{tabular}




\begin{tabular}{|c|c|c|}
\hline Nom des muscles & Figures & Pages \\
\hline Grand palmaire $\ldots \ldots \ldots \ldots \ldots \ldots \ldots$ & 15 & 120 \\
\hline Grand psoas $\ldots \ldots \ldots \ldots \ldots \ldots \ldots \ldots$ & 9 & 114 \\
\hline 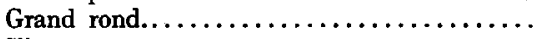 & $3,14,15$ & 118 \\
\hline Iliaque $\ldots \ldots \ldots \ldots \ldots \ldots \ldots \ldots \ldots$ & 9,13 & 116 \\
\hline Intertransversaire du cou............... & $4,5,6$ & 112 \\
\hline Jumeaux du bassin .................. & 12,13 & 116 \\
\hline Jumeau externe de la jambe ............ & 10,12 & 116 \\
\hline Jumeau interne de la jambe.............. & $10,11,12$ & 116 \\
\hline 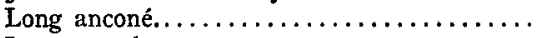 & 14,15 & 118 \\
\hline 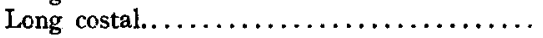 & 5,6 & 112 \\
\hline Long du cou $\ldots \ldots \ldots \ldots \ldots \ldots \ldots \ldots$ & 1,7 & 111 \\
\hline 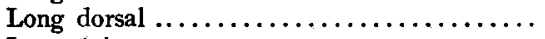 & $3,4,5,6,8$ & 112 \\
\hline 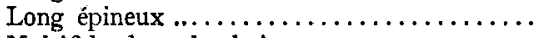 & 5,6 & 112 \\
\hline Multifide dorso-lombaire ............... & 6 & 112 \\
\hline Oblique externe de l'abdomen ........... & $2,3,5,8$ & 113 \\
\hline Oblique interne de l'abdomen............ & & 113 \\
\hline Obturateur externe et obturateur interne.... & 13 & 116 \\
\hline 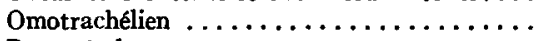 & 1,2 & 109 \\
\hline 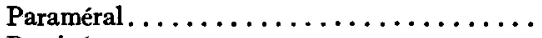 & 10 & 114 \\
\hline Pectiné $\ldots \ldots \ldots \ldots \ldots \ldots \ldots \ldots$ & 13 & 115 \\
\hline Pectoral ascendant . . . . . . . . . . & 1 & 110 \\
\hline 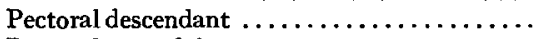 & 1,2 & 109 \\
\hline 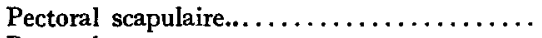 & $1,2,3$ & 110 \\
\hline Pectoral transverse.$\ldots \ldots \ldots \ldots \ldots \ldots$. & 1 & 109 \\
\hline Perforant (membre antérieur) ........... & 15 & 120 \\
\hline Perforé (membre antérieur) ............. & 15 & 120 \\
\hline Perforé (membre postérieur) . . . . . . . . . . & 11,12 & 117 \\
\hline Péroniers $\ldots \ldots \ldots \ldots \ldots \ldots \ldots \ldots \ldots \ldots \ldots$ & 10,12 & 117 \\
\hline 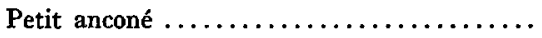 & 14 & 118 \\
\hline Petit complexus................. & 5 & 110 \\
\hline Petit dentelé antérieur................ & 4,8 & 110 \\
\hline Petit dentelé postérieur ................ & 4,8 & 110 \\
\hline Petit droit antérieur de la tête $\ldots \ldots \ldots \ldots$. & $4,5,6,7$ & 111 \\
\hline Petit droit latéral de la tête............ & $4,5,6$ & 111 \\
\hline Petit droit postérieur de la tête .......... & 6 & 111 \\
\hline Petit oblique de la tête..$\ldots \ldots \ldots \ldots \ldots$. & 6 & 111 \\
\hline 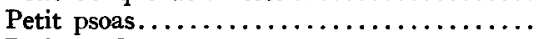 & 9 & 113 \\
\hline Petit rond $\ldots \ldots \ldots \ldots \ldots \ldots \ldots \ldots \ldots$ & 14 & 118 \\
\hline Poplité $\ldots \ldots \ldots \ldots \ldots \ldots \ldots \ldots \ldots \ldots \ldots \ldots \ldots \ldots \ldots \ldots$ & 12 & 117 \\
\hline 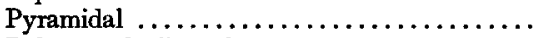 & 12 & 116 \\
\hline Releveur de l'épaule................. & 3 & 109 \\
\hline 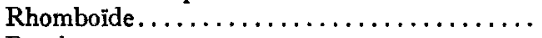 & 3 & 109 \\
\hline 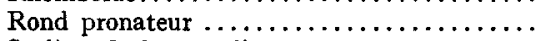 & 15 & 120 \\
\hline Scalène de la première côte...$\ldots \ldots \ldots \ldots$ & $4,5,7$ & 112 \\
\hline Scalène supra-costal $\ldots \ldots \ldots \ldots \ldots \ldots$ & $3,4,5,6,8$ & 112 \\
\hline Scansorius $\ldots \ldots \ldots \ldots \ldots \ldots \ldots \ldots \ldots$ & 12,13 & 115 \\
\hline 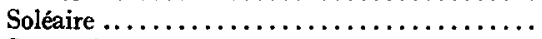 & 11,12 & 117 \\
\hline 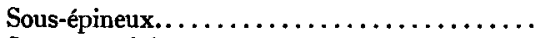 & 3,14 & 118 \\
\hline Sous-scapulaire $\ldots \ldots \ldots \ldots \ldots \ldots \ldots \ldots$ & 15 & 118 \\
\hline Splénius $\ldots \ldots \ldots \ldots \ldots \ldots \ldots \ldots \ldots$ & $3,4,7$ & 110 \\
\hline Stermo-céphalique .................. & 1,2 & 108 \\
\hline Sterno-hyoïdien et sterno-thyroïdien........ & $1,2,3,4,5$ & 108 \\
\hline Sus-épineux....................... & $2,3,14,15$ & 118 \\
\hline Tenseur du fascia lata .................. & 10,11 & 114 \\
\hline 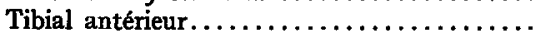 & $10,11,12$ & 117 \\
\hline Transverse de l'abdomen................ & 8,9 & 113 \\
\hline Transversaire du cou................. & 5,6 & 111 \\
\hline
\end{tabular}




\begin{tabular}{|c|c|c|}
\hline Nom des muscles & Figures & Pages \\
\hline 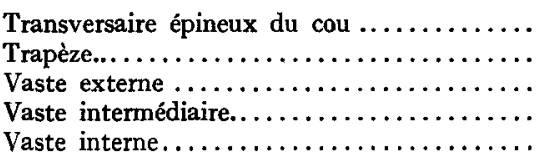 & $\begin{array}{l}6 \\
1,2 \\
10 \\
11,12 \\
11\end{array}$ & $\begin{array}{l}112 \\
109 \\
115 \\
115 \\
115\end{array}$ \\
\hline
\end{tabular}

\section{SUMMARY}

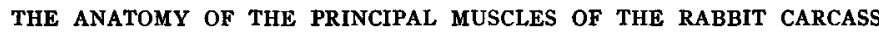

A thorough method of dissection was worked out in view of further investigations on the growth and development of the rabbit.

The bone muscles have been listed, with an operative index as an atlas describing the principal muscles of the carcass, following the pattern of dissection and illustrates with anatomical plates.

\section{RÉFÉRENCES BIBLIOGRAPHIQUES}

AlezaIs H., Igor. Contribution a la myologie des Rongeurs. Alcan, Paris.

CRAIGIE E. H., 1960. Practical anatomy of the Rabbit. University of Toronto Press. Toronto.

Dobberstein, Hoffmann, 1965. Lehrbuch der vergleichenden Anatomie der Haustiere. S. Hirzel Verlag Leipzig.

Faisan J. A., I95I. Contribution de l'étude de la myologie du Lapin. Thèse Doct. Vét., Lyon.

GerHARDT U., Igog. Das Kaninchen. Verlag von Werner Klinkhardt, Leipzig.

HAACK K., 1903. Vergleichende untersuchungen über die muskulatur der Gliedmassen und des Stammes bei der Katze, dem Hasen und Kaninchen. Inaugural dissertation Univ. Bern.

KraUSE 1884. Die Anatomie des Kaninchens in topographischer und operativer Hinsicht. Leipzig.

Montane L., Bourdelle E., BREssou C., 1937. Anatomie régionale des animaux domestiques. Baillière et fils, Paris.

Nickel R., Schumer A., SeIferde E., 1954. Lehrbuch der Anatomie der Haustiere. P. Parey, Berlin. SchwARZE, 1960. Kompendium der Veterinär Anatomie. Veb. Gustav. Fischer Verlag, Jena.

Sisson S., Grossman J. D., 1953. The anatomy of the domestic animals. W. B. Saunders Company. 